\title{
QCD in One Dimension at Nonzero Chemical Potential
}

\author{
L. Ravagli \\ Cyclotron Institute and Physics Department TEXAS AEGM University, College Station, Texas 77843-3366, USA \\ J.J.M. Verbaarschot \\ Niels Bohr International Academy, Blegdamsvej 17, DK-2100, Copenhagen Ø, Denmark, \\ Niels Bohr Institute, Blegdamsvej 17, DK-2100, Copenhagen Ø, Denmark and \\ Department of Physics and Astronomy, SUNY, Stony Brook, New York 11794, USA
}

\begin{abstract}
Using an integration formula recently derived by Conrey, Farmer and Zirnbauer, we calculate the expectation value of the phase factor of the fermion determinant for the staggered lattice QCD action in one dimension. We show that the chemical potential can be absorbed into the quark masses; the theory is in the same chiral symmetry class as QCD in three dimensions at zero chemical potential. In the limit of a large number of colors and fixed number of lattice points, chiral symmetry is broken spontaneously, and our results are in agreement with expressions based on a chiral Lagrangian. In this limit, the eigenvalues of the Dirac operator are correlated according to random matrix theory for QCD in three dimensions. The discontinuity of the chiral condensate is due to an alternative to the Banks-Casher formula recently discovered for QCD in four dimensions at nonzero chemical potential. The effect of temperature on the average phase factor is discussed in a schematic random matrix model.
\end{abstract}

\section{INTRODUCTION}

In spite of significant recent progress, QCD at nonzero chemical potential remains a notoriously hard problem [1, 2, 3]. In particular, first principle nonperturbative results at low temperature are absent because the phase of the fermion determinant invalidates probabilistic methods to evaluate the partition function. This problem is known as the sign problem. The sign problem is particularly severe if the phase of the fermion determinant results in a different free energy, i.e.

$$
\frac{\left\langle\operatorname{det}^{N_{f}} D\right\rangle}{\left\langle\left|\operatorname{det}^{N_{f}} D\right|\right\rangle}=e^{V\left(F_{\mathrm{pq}}-F_{N_{f}}\right)} \quad \text { with } \quad F_{N_{f}}>F_{\mathrm{pq}} .
$$

If the phase quenched free energy, $F_{\mathrm{pq}}$ differs from the free energy of QCD with $N_{f}$ flavors, $F_{N_{f}}$, the number of required gauge field configurations grows exponentially with the volume. The ratio defined in equation (10) can also be interpreted as the average phase factor of the fermion determinant [4, [5]

$$
\left\langle e^{i N_{f} \theta}\right\rangle_{\mathrm{pq}}=\frac{\left\langle e^{i N_{f} \theta}\left|\operatorname{det}^{N_{f}} D\right|\right\rangle}{\left\langle\left|\operatorname{det}^{N_{f}} D\right|\right\rangle} .
$$

Alternatively, one can define the average phase factor with respect to the full QCD partition function [4, 5]

$$
\left\langle e^{2 i \theta}\right\rangle_{N_{f}}=\frac{\left\langle e^{2 i \theta} \operatorname{det}^{N_{f}} D\right\rangle}{\left\langle\operatorname{det}^{N_{f}} D\right\rangle},
$$

which has also been used in the literature $[4,5,6,67,8,4,10,11,12]$ as a measure for the severity of the sign problem.

When the chemical potential is sufficiently small, the average phase factor can be studied by means of chiral perturbation theory [4, 5]. Recently, the average phase factor was analyzed in the microscopic domain of QCD [13, 14], where only the constant fields in the chiral Lagrangian contribute to the mass and chemical potential dependence of the partition function. It was found that the sign problem is not serious for $\mu<m_{\pi} / 2$. For $\mu>m_{\pi} / 2$ the chiral condensate of the phase quenched theory rotates into a pion condensate resulting in a free energy that is different from the full theory and a severe sign problem.

In this paper we study the sign problem for Euclidean QCD in one dimension. In one dimension, the only effect of the gauge field is in the boundary conditions, and lattice QCD simplifies to a matrix integral. A general formula for exactly this type of integrals was recently derived by Conrey, Farmer and Zirnbauer [15]. Using what we will call the CFZ formula, exact analytical results for the one dimensional QCD partition function and the average phase factor will be obtained.

The study of QCD in one dimension at finite chemical potential has had a long history. One reason to study this model is that it is an effective model for the large $N_{c}$ limit of strong coupling QCD [16, 17, 18, 19, 20, 21]. Among 
others, it has been successfully used to explain [22, 23, 24] puzzling lattice results for quenched QCD at nonzero chemical potential. More recently, one dimensional QCD was used to study the complex zeros of the partition function [1, 25, 26].

The sign problem of QCD at nonzero chemical potential arises because of the nonhermiticity of the Dirac operator. QCD in one dimension is not truly nonhermitean: Instead of being scattered in a two-dimensional domain of the complex plane [27], the eigenvalues of the Dirac operator are localized on an ellipse in the complex plane 6, 22]. Another manifestation of the mild nonhermiticity is that the chemical potential can be absorbed into the quark masses. For each flavor in one dimension with quark mass $m$, we can associate flavors with mass $m+\mu$ and $-m+\mu$. In the large $N_{c}$ limit, this results in spontaneous chiral symmetry breaking according to $U\left(2 N_{f}\right) \rightarrow U\left(N_{f}\right) \times U\left(N_{f}\right)$. Therefore, QCD in one dimension is in the same chiral symmetry class as QCD in three dimensions. The microscopic limit of QCD in one dimension is equivalent to the microscopic limit of QCD in three dimensions [28]. Another peculiarity of QCD in one dimension is that, already in the free theory, the eigenvalues near zero are spaced inversely proportional to the volume. Therefore, in the thermodynamic limit (i.e. at zero temperature) the chiral condensate is discontinuous across the imaginary axis. However, this type of symmetry breaking is not a collective phenomenon, and there are no associated Goldstone bosons. It is reminiscent to the alternative to Goldstone's theorem proposed by McKane and Stone [29]. Spontaneous symmetry breaking with Goldstone bosons takes place for a fixed number of lattice points in the limit $N_{c} \rightarrow \infty$.

A chemical potential excites color singlet excitations with baryon charge $q_{k}$ and mass $M_{k}$ when $q_{k} \mu>M_{k}$. This is also the case for one-dimensional QCD. For gauge group $U\left(N_{c}\right)$, the only color singlet excitations are mesons that are uncharged with respect to the chemical potential, whereas for $S U\left(N_{c}\right)$ gauge group we have both neutral mesonic and charged baryonic color singlet excitations. The complex conjugate of the fermion determinant can be interpreted in terms of conjugate quarks [23, 30] which have a baryon charge that is opposite to that of regular quarks. Therefore, both for $U\left(N_{c}\right)$ and $S U\left(N_{c}\right)$, the phase quenched partition function, where the fermion determinant has been replaced by its absolute value, has charged mesonic excitations made out of quarks and conjugate anti-quarks. This results in a phase transition at $\mu=\mu_{c}=m_{\pi} / 2$. The $S U\left(N_{c}\right)$ theory with $N_{f}$ flavors has only charged baryonic excitations and will have a phase transition at $\mu=\mu_{c}=m_{B} / N_{c}$. In one dimension it turns out that the pion and the baryon have the same mass per quark number so that the critical chemical potential of the phase quenched $U\left(N_{c}\right)$ theory and the $S U\left(N_{c}\right)$ theory is the same.

For the $U\left(N_{c}\right)$ partition function we expect the sign problem to be severe when $\mu>\mu_{c}$ because the phase quenched partition function has a phase transition at $\mu=\mu_{c}$ whereas the normal theory remains in the same phase. For $S U\left(N_{c}\right)$, both the normal partition function and the phase quenched partition function do have a phase transition to a phase of free quarks at $\mu=\mu_{c}$ and have the same free energy not only for $\mu<\mu_{c}$ but also for $\mu>\mu_{c}$. Therefore, in one dimension there is no severe sign problem for $S U\left(N_{c}\right)$ QCD.

Because one dimensional QCD with gauge group $U\left(N_{c}\right)$ does not have a phase transition, the chiral condensate in the thermodynamic limit or large $N_{c}$ limit is discontinuous at $m=0$, independent of the value of the chemical potential. Because the Dirac eigenvalues are located on an ellipse for $\mu \neq 0$, this discontinuity cannot be related to the Dirac spectrum by means of the Banks-Casher formula. In [31] a different mechanism to explain the discontinuity was discovered. It was found that an oscillating contribution to the spectral density with an amplitude that diverges exponentially with the volume, is responsible for the discontinuity of the chiral condensate. We will show that a similar mechanism is at work for $U\left(N_{c}\right)$ gauge theory in one dimension.

Lattice QCD in one dimension will be introduced in section II, where we also discuss its continuum limit, mean field results and the Conrey-Farmer-Zirnbauer formula. In section III we will evaluate the $U\left(N_{c}\right)$ partition function and its phase quenched version. The average phase factor for $U\left(N_{c}\right)$ is calculated in section IV. In section V we study one dimensional QCD with $S U\left(N_{c}\right)$ as gauge group and evaluate the regular and phase quenched partition functions and the average phase factor. The effect of temperature will be illustrated with results from a schematic random matrix model in section VI. The connection with the microscopic domain of QCD in three dimensions is discussed in section VII, and the Dirac spectrum and its relation with chiral symmetry breaking is analyzed in section VIII. Concluding remarks are made in section IX. 


\section{QCD IN ONE DIMENSION}

\section{A. Lattice QCD}

The staggered lattice QCD Dirac operator in one dimension is given by

$$
D=\left(\begin{array}{cccc}
m I & e^{\mu} U_{12} / 2 & \ldots & e^{-\mu} U_{n 1}^{\dagger} / 2 \\
-e^{-\mu} U_{12}^{\dagger} / 2 & m I & \ldots & 0 \\
\vdots & & & \vdots \\
0 & \ldots & m I & e^{\mu} U_{n-1 n} / 2 \\
-e^{\mu} U_{n 1} / 2 & \ldots & -e^{-\mu} U_{n-1 n}^{\dagger} / 2 & m I
\end{array}\right)
$$

We have used anti-periodic boundary conditions and the gauge fields on the links are taken to be in $U\left(N_{c}\right)$ or $S U\left(N_{c}\right)$. The chemical potential $\mu$ is an imaginary vector field introduced according to the Hasenfratz-Karsch prescription 32 . In general, the lattice QCD partition function for $N_{f}$ flavors with mass $m$ is given by

$$
Z_{N_{f}}\left(\mu_{c}, \mu\right)=\int \prod_{l} d U_{l} \operatorname{det}^{N_{f}} D e^{-S_{\mathrm{YM}} / g^{2}}
$$

where the product is over the links and $S_{\mathrm{YM}}$ is the Yang-Mills action. In one dimension, the partition function simplifies substantially. First, there is no Yang-Mills action, and second, by unitary transformations the integral over the links can be reduced to a single integral. In the gauge where all gauge fields except $U_{n 1} \equiv U$ are equal the unity, the fermion determinant reduces to [24]

$$
\operatorname{det} D=2^{-n N_{c}} \operatorname{det}\left[e^{n \mu_{c}}+e^{-n \mu_{c}}+e^{n \mu} U+e^{-n \mu} U^{\dagger}\right]
$$

with $\mu_{c}$ given by

$$
\mu_{c}=\sinh ^{-1} m
$$

This value will turn out to be the critical value of the chemical potential. From now on, the overall factor $2^{-n N_{c}}$ will be absorbed into the normalization of the partition function. To avoid sign factors, $n$ is taken to be even throughout this paper.

The standard method to evaluate the QCD partition function in one dimension is to use the eigenvalues of $U$ as integration variables [6, 16, 17, 18, 24]. For example, one finds this way that the result for $N_{f}=1$ with $U\left(N_{c}\right)$ as gauge group is given by [24]

$$
Z_{N_{f}=1}\left(\mu_{c}, \mu\right)=\int_{U\left(N_{c}\right)} d U \operatorname{det} D=\frac{\sinh \left(\left(N_{c}+1\right) n \mu_{c}\right)}{\sinh \left(n \mu_{c}\right)}
$$

For most partition functions that are considered in this paper it is not possible to obtain analytical results by means of this method. Instead we will use powerful integration formulas for unitary integrals that were recently derived in [15]. These integrals are based on the color-flavor transformation [33] which has also been applied to lattice QCD with baryons in the canonical ensemble [34, 35].

\section{B. Continuum Theory}

The continuum limit of the staggered lattice action is given by

$$
D_{\text {cont }}=\left(\begin{array}{cc}
m & \partial_{0}+i A_{0}+\mu \\
\partial_{0}+i A_{0}+\mu & m
\end{array}\right),
$$

where $A_{0}$ is a Hermitean $N_{c} \times N_{c}$ matrix and the off-diagonal blocks connect even and odd lattice sites. What is special in one dimension is that the off-diagonal blocks are identical. The eigenvalue equation $\left(\partial_{0}+i A_{0}\right) \psi_{k}=i u_{k} \psi_{k}$ is solved by

$$
\psi=P e^{-i \int_{0}^{t} A_{0} d t} \chi_{0} \quad \text { with } \quad \partial_{0} \chi_{0}=i E \chi_{0},
$$


with $P e$ the path ordered exponent. Nontrivial eigenvalues are obtained by imposing boundary conditions on $\psi$. At nonzero chemical potential the eigenvalues of the Dirac operator are given by

$$
\lambda_{k}=m \pm\left(i u_{k}+\mu\right) \quad \text { with } \quad u_{k} \in \mathbb{R} .
$$

This is very different from QCD with $d \geq 2$ where the eigenvalues of the QCD Dirac operator at $\mu \neq 0$ are scattered in the complex plane. Also the eigenvalues of the one dimensional staggered lattice Dirac at nonzero chemical potential are localized on a curve in the complex plane.

Another consequence of the structure of the Dirac operator (9) is that the fermion determinant can be rewritten as

$$
\operatorname{det} D_{\text {cont }}=\operatorname{det}\left(\partial_{0}+i A_{0}+\mu+m\right) \operatorname{det}\left(\partial_{0}+i A_{0}+\mu-m\right)
$$

which can be interpreted as a two-flavor partition function with masses $m+\mu$ and $-m+\mu$. This Dirac operator has the same structure as QCD in three dimensions: In the large $N_{c}$ limit, chiral symmetry is broken spontaneously according to $U(2) \rightarrow U(1) \times U(1)$ with the squared Goldstone masses proportional to the difference of the positive and negative quark masses, i.e. $m_{\pi}^{2} \sim m-(-m)=2 m$. At low energy, these Goldstone modes interact according to a chiral Lagrangian determined by the pattern of chiral symmetry breaking. In the large $N_{c}$ limit and $m_{\pi} \beta \ll 1$ (with $\beta$ the length of the one-dimensional box) the QCD partition function in one dimension is therefore equivalent to the low-energy limit of QCD in three dimensions. In section VII this will be worked out explicitly for the microscopic limit of the partition function. This is the limit

$$
\rho(0) \rightarrow \infty \text { with } m \pi \rho(0)=\text { fixed and } \mu \pi \rho(0)=\text { fixed, }
$$

where $\rho(0)$ is density of the eigenvalues, or their projections onto the imaginary axis, close to zero. Since $\rho(0)=n N_{c} / 2 \pi$ (see section VIII), this is the limit $n N_{c} \rightarrow \infty$ with $n N_{c} m$ and $n N_{c} \mu$ fixed. When we use the term microscopic limit, we always mean the universal microscopic limit. This is the limit (13) associated with the formation of Goldstone bosons, i.e. the limit $N_{c} \rightarrow \infty$ at fixed $n$.

\section{Mean Field Limit for Large $N_{c}$}

In the large $N_{c}$ limit, chiral symmetry is broken spontaneously even in one dimension, so that its low-energy limit is a theory of Goldstone bosons. In this section we give general arguments that determine the chemical potential dependence of the partition function in the microscopic domain where the Compton wavelength of the Goldstone bosons is much larger than $\beta$.

As was argued in [4, 5], in the microscopic domain, the mean field limit of the partition function is given by

$$
Z=J\left(\prod_{k} \frac{1}{m_{\pi}(\mu)}\right) e^{-V F}
$$

where $J$ is the value of the integration measure at the saddle point, $F$ is the free energy, $V$ is the space time volume, and $m_{\pi}$ are the masses of the Goldstone bosons. Let us apply this result to the average phase factor in one dimension. For $\mu<\mu_{c}$, in the limit $T \rightarrow 0$, only the vacuum state contributes to the partition function, so that the free energy is independent of $\mu$. For quark mass $m$, the equivalent $\mathrm{QCD}_{3}$ mass matrix of the Dirac operator $D$ in (12) is given by $\operatorname{diag}(-m+\mu, m+\mu)$, whereas the hermitean conjugate Dirac operator $D^{\dagger}$, has $\operatorname{diag}(-m-\mu, m-\mu)$ as equivalent $\mathrm{QCD}_{3}$ mass matrix. For $N_{f}$ flavors, the average phase factor is the partition function with $N_{f}+1$ fermionic quarks and one conjugate bosonic quark. We thus have $2\left(N_{f}+1\right)^{2}$ Goldstone bosons made out of two fermionic quarks with squared mass $m_{\pi}^{2}=2 m G$ (with $G$ a constant), $4\left(N_{f}+1\right)$ fermionic Goldstone modes, half of them with squared mass $m_{\pi}^{2}=2(m-\mu) G$, and the other half with squared mass $m_{\pi}^{2}=2(m+\mu) G$. Finally, we have 2 Goldstone bosons composed out of two bosonic quarks with squared mass equal to $m_{\pi}^{2}=2 m G$. The fermionic partition function $Z_{N_{f}}$ has $2 N_{f}^{2}$ Goldstone bosons all with squared mass $m_{\pi}^{2}=2 m G$. Using (14) we thus find

$$
\left\langle e^{2 i \theta}\right\rangle_{N_{f}}=\frac{Z_{N_{f}+1 \mid 1^{*}}\left(\mu_{c}, \mu\right)}{Z_{N_{f}}\left(\mu_{c}, \mu\right)}=\left(1-\frac{\mu^{2}}{m^{2}}\right)^{N_{f}+1} \quad \text { for } \quad \mu<\mu_{c} .
$$

For phase quenched partition functions with $N_{f}$ flavors and $N_{f}$ conjugate flavors, the equivalent $\mathrm{QCD}_{3}$ symmetry breaking pattern is $U\left(4 N_{f}\right) /\left(U\left(2 N_{f}\right) \times U\left(2 N_{f}\right)\right)$, so that the total number of Goldstone bosons is equal to $8 N_{f}^{2}$. Of these, $4 N_{f}^{2}$ Goldstone bosons have squared mass $m_{\pi}^{2}=2 m G$, half made out of two quarks and the other half out of two 
conjugate quarks. The other $4 N_{f}^{2}$ Goldstone bosons are composed out of a quark and a conjugate anti-quark quark, half with squared mass $m_{\pi}^{2}=2(m-\mu) G$ and the other half with squared mass $m_{\pi}^{2}=2(m+\mu) G$. All equivalent $\mathrm{QCD}_{3}$ Goldstone bosons of the full QCD partition function with $2 N_{f}$ flavors have a squared mass equal to $m_{\pi}^{2}=2 m G$. For $\mu<\mu_{c}$ the free energy of the phase quenched partition function and the full QCD partition function is the same so that, using (14), the phase quenched average phase factor is given by

$$
\left\langle e^{2 i \theta}\right\rangle_{N_{f}+N_{f}^{*}}=\frac{Z_{N_{f}+1+\left(N_{f}-1\right)^{*}}\left(\mu_{c}, \mu\right)}{Z_{2 N_{f}}\left(\mu_{c}, \mu\right)}=\left(1-\frac{\mu^{2}}{m^{2}}\right)^{N_{f}^{2}} \quad \text { for } \quad \mu<\mu_{c} .
$$

Below we will show that the results derived in this section also follow from the zero temperature microscopic limit of the exact evaluation of the partition function.

\section{The Conrey-Farmer-Zirnbauer formula}

Exactly the integrals that are required for the evaluation of the average phase factor of QCD at nonzero chemical potential in one dimension were studied in a recent paper by Conrey, Farmer and Zirnbauer 15. They considered the partition function

$$
Z\left(\left\{\psi_{k}, \phi_{k}\right\}\right)=\int_{U\left(N_{c}\right)} d U \prod_{j=1}^{p} \frac{\operatorname{det}\left(1-e^{\psi_{j}} U\right)}{\operatorname{det}\left(1-e^{\phi_{j}} U\right)} \prod_{l=p+1}^{p+q} \frac{\operatorname{det}\left(1-e^{-\psi_{l}} U^{\dagger}\right)}{\operatorname{det}\left(1-e^{-\phi_{l}} U^{\dagger}\right)},
$$

with $d U$ the Haar measure of $U\left(N_{c}\right)$ and $\psi_{k}, \phi_{k}$ complex parameters with $\operatorname{Re}\left(\phi_{j}\right)<0<\operatorname{Re}\left(\phi_{l}\right)$. Using the color flavor transformation [33] and Howe's theory of supersymmetric dual pairs, they derived the following formula

$$
Z\left(\left\{\psi_{k}, \phi_{k}\right\}\right)=\sum_{\pi \in S_{p+q} /\left(S_{p} \times S_{q}\right)} \prod_{l+1}^{p+q} e^{N_{c}\left(\pi\left(\psi_{l}\right)-\psi_{l}\right)} \prod_{j=1}^{p} \frac{\left(1-e^{\phi_{j}-\pi\left(\psi_{l}\right)}\right)\left(1-e^{\pi\left(\psi_{j}\right)-\phi_{l}}\right)}{\left(1-e^{\pi\left(\psi_{j}\right)-\pi\left(\psi_{l}\right)}\right)\left(1-e^{\phi_{j}-\phi_{l}}\right)}
$$

The sum is over permutations in $S_{p+q} /\left(S_{p} \times S_{q}\right)$ that interchange any of the $\psi_{1}, \cdots, \psi_{p}$ with any of the $\psi_{p+1}, \cdots, \psi_{p+q}$.

Partition functions with an unequal number of bosonic and fermionic determinants can be obtained from special limits of (18). In the case of only fermionic determinants, an equivalent expression was first obtained in [36, 37]. Expressions for degenerate parameters can be derived by carefully taking limits of the above formula.

Unitary matrix integrals can also be calculated by using an eigenvalue representation of the unitary matrices. The integrals we are interested in are of the form

$$
Z=\int_{U\left(N_{c}\right)} d U \prod_{k} F\left(e^{i \theta_{k}}\right)
$$

where $\exp \left(i \theta_{k}\right)$ are the eigenvalues of $U$. Using an eigenvalue representation of the unitary matrices, the orthogonal polynomial method can be used to express $Z$ as [17]

$$
Z=\operatorname{det}\left(B_{k-l}\right)_{k, l=0, \cdots, N_{c}-1},
$$

where

$$
B_{k}=\frac{1}{2 \pi} \int_{-\pi}^{\pi} d \theta e^{i k \theta} F\left(\left\{e^{i \theta}\right\}\right) .
$$

This is the method that was used in the literature on one-dimensional QCD prior to this paper [6, 16, 17, 18, 24]. In a few cases the determinant in (20) could be evaluated explicitly resulting in expressions that are similar to those derived directly from the CFZ-formula. We have used (20) to numerically check the results obtained by means of the CFZ-formula.

\section{EXACT EVALUATION OF THE ONE-DIMENSIONAL $U\left(N_{c}\right)$ PARTITION FUNCTION}

\section{A. Partition Function for arbitrary $N_{f}$}

In this section we evaluate the one-dimensional $U\left(N_{c}\right)$ QCD partition function for $N_{f}$ flavors. 
To apply the CFZ formula (18) we rewrite the determinant (6) as

$$
\operatorname{det} D=e^{\mu_{c} n N_{c}} \operatorname{det}\left(1+e^{n\left(\mu-\mu_{c}\right)} U\right) \operatorname{det}\left(1+e^{n\left(-\mu-\mu_{c}\right)} U^{\dagger}\right) .
$$

For arbitrary $N_{f}$ we then find the remarkably simple answer

$$
Z_{N_{f}}\left(\mu_{c}, \mu\right) \equiv \int_{U\left(N_{c}\right)} d U \operatorname{det}^{N_{f}} D=\sum_{\sigma \in S_{2_{f}} / S_{N_{f}} \times S_{N_{f}}} \prod_{k=1}^{N_{f}} \prod_{l=1}^{N_{f}} \frac{e^{N_{c} m_{\sigma(+k)}}}{1-\exp \left(m_{\sigma(-l)}-m_{\sigma(+k)}\right)},
$$

with

$$
m_{-k}=-n \mu_{c k} \quad \text { and } \quad m_{+k}=n \mu_{c k} .
$$

The sum is over all permutations that interchange positive and negative masses. Notice that the $\mu$-dependence has canceled from this expression. This also follows from an expansion of the determinant in powers of $U$ and $U^{\dagger}$. Only terms with an equal number of factors $U$ and factors $U^{\dagger}$ are non-vanishing.

Although the result for degenerate positive and negative masses can be obtained by carefully taking limits of (23), it is simpler to start from a different representation [36, 37] of partition function (23) given by

$$
Z_{N_{f}}\left(\mu_{c}, \mu\right)=\frac{1}{\prod_{1 \leq k<l \leq 2 N_{f}}\left(e^{M_{l}}-e^{M_{k}}\right)}\left|\begin{array}{ccccccc}
1 & e^{m_{-1}} & \cdots & e^{\left(N_{f}-1\right) m_{-1}} & e^{\left(N_{c}+N_{f}\right) m_{-1}} & \cdots & e^{\left(N_{c}+2 N_{f}-1\right) m_{-1}} \\
\vdots & \vdots & & \vdots & \vdots & \vdots \\
1 & e^{m_{-N_{f}}} & \cdots & e^{\left(N_{f}-1\right) m_{-} N_{f}} & e^{\left(N_{c}+N_{f}\right) m_{-} N_{f}} & \cdots & e^{\left(N_{c}+2 N_{f}-1\right) m_{-} N_{f}} \\
1 & e^{m_{+1}} & \cdots & e^{\left(N_{f}-1\right) m_{+1}} & e^{\left(N_{c}+N_{f}\right) m_{+1}} & \cdots & e^{\left(N_{c}+2 N_{f}-1\right) m_{+}} \\
\vdots & \vdots & & \vdots & \vdots & \vdots \\
1 & e^{m_{+N_{f}}} & \cdots & e^{\left(N_{f}-1\right) m_{+} N_{f}} & e^{\left(N_{c}+N_{f}\right) m_{+} N_{f}} & \cdots & e^{\left(N_{c}+2 N_{f}-1\right) m_{+} N_{f}}
\end{array}\right| .
$$

For convenience we have introduced the mass matrix $M_{k}=\left(m_{11}, \cdots, m_{-N_{f}}, m_{+1}, \cdots, m_{+} N_{f}\right)$. To obtain an expression for degenerate masses, we Taylor expand the exponential functions $\exp \left(m_{+k}\right)$ and $\exp \left(m_{-k}\right)$ to order $N_{f}-1$ about $\exp \left(-n \mu_{c}\right)$ and $\exp \left(n \mu_{c}\right)$, respectively, and write the resulting matrix as the product of two matrices, one containing the Taylor coefficients, and the other containing powers of the expansion parameters. The determinant of the second matrix can be written as a Vandermonde determinant which cancels against part of the prefactor in (25). Our final expression for the partition function with degenerate masses is given by

$$
\begin{aligned}
& Z_{N_{f}}\left(\mu_{c}, \mu\right)=\frac{1}{\left(\prod_{k=0}^{N_{f}-1} k !\right)^{2}} \frac{1}{\left(e^{n \mu_{c}}-e^{-n \mu_{c}}\right)^{N_{f}^{2}}} \\
& \times\left|\begin{array}{cccccc}
1 & \cdots & 0 & 1 & \cdots & 0 \\
e^{-n \mu_{c}} & \cdots & e^{-n \mu_{c}} & e^{n \mu_{c}} & \cdots & e^{n \mu_{c}} \\
e^{-2 n \mu_{c}} & \cdots & \delta_{-}^{N_{f}-1} e^{-2 n \mu_{c}} & e^{2 n \mu_{c}} & \cdots & \delta_{+}^{N_{f}-1} e^{2 n \mu_{c}} \\
\vdots & & : & & \vdots & \\
e^{-n\left(N_{f}-1\right) \mu_{c}} & \cdots & \delta_{-}^{N_{f}-1} e^{-n\left(N_{f}-1\right) \mu_{c}} & e^{n\left(N_{f}-1\right) \mu_{c}} & \cdots & \delta_{+}^{N_{f}-1} e^{n\left(N_{f}-1\right) \mu_{c}} \\
e^{-n\left(N_{c}+N_{f}\right) \mu_{c}} & \cdots & \delta_{-}^{N_{f}-1} e^{-n\left(N_{c}+N_{f}\right) \mu_{c}} & e^{n\left(N_{c}+N_{f}\right) \mu_{c}} & \cdots & \delta_{+}^{N_{f}-1} e^{n\left(N_{c}+N_{f}\right) \mu_{c}} \\
\vdots & & : & & \vdots & \\
e^{-n\left(N_{c}+2 N_{f}-1\right) \mu_{c}} & \cdots & \delta_{-}^{N_{f}-1} e^{-n\left(N_{c}+2 N_{f}-1\right) \mu_{c}} & e^{n\left(N_{c}+2 N_{f}-1\right) \mu_{c}} & \cdots & \delta_{+}^{N_{f}-1} e^{n\left(N_{c}+2 N_{f}-1\right) \mu_{c}}
\end{array}\right|,
\end{aligned}
$$

with

$$
\delta_{-}=\frac{d}{d\left(-n \mu_{c}\right)}, \quad \delta_{+}=\frac{d}{d\left(n \mu_{c}\right)}
$$

The $k^{\prime}$ th column (with $k \leq N_{f}$ ) is given by the $\delta_{-}^{(k-1)}$ derivative of the first column, and the $N_{f}+k^{\prime}$ th column is given by the $\delta_{+}^{(k-1)}$ derivative of the $N_{f}$ 'th column.

From this result one can easily derive explicit expressions for small values of $N_{f}$. The partition function for $N_{f}=2$ reads

$$
Z_{N_{f}=2}\left(\mu_{c}, \mu\right)=\frac{\left(e^{n\left(N_{c}+2\right) \mu_{c}}-e^{-n\left(N_{c}+2\right) \mu_{c}}\right)^{2}}{\left(e^{n \mu_{c}}-e^{-n \mu_{c}}\right)^{4}}-\frac{\left(N_{c}+2\right)^{2}}{\left(e^{n \mu_{c}}-e^{-n \mu_{c}}\right)^{2}} .
$$


The microscopic limit of this partition function is given by

$$
Z_{N_{f}=2}^{\text {micro }}\left(\mu_{c}, \mu\right)=\frac{\left(e^{N_{c} \mu_{c}}-e^{-N_{c} \mu_{c}}\right)^{2}}{16 \mu_{c}^{4}} .
$$

For $N_{f}=3$ the partition function is given by:

$$
\begin{aligned}
Z_{N_{f}=3}\left(\mu_{c}, \mu\right)= & \frac{\left(e^{n\left(N_{c}+3\right) \mu_{c}}-e^{-n\left(N_{c}+3\right) \mu_{c}}\right)^{3}}{\left(e^{n \mu_{c}}-e^{-n \mu_{c}}\right)^{9}}-\left(N_{c}+3\right)^{3} \frac{\left(e^{n\left(N_{c}+1\right) \mu_{c}}-e^{-n\left(N_{c}+1\right) \mu_{c}}\right)}{\left(e^{n \mu_{c}}-e^{-n \mu_{c}}\right)^{7}} \\
& +N_{c}\left(N_{c}+2\right)^{2} \frac{\left(e^{n\left(N_{c}+3\right) \mu_{c}}-e^{-n\left(N_{c}+3\right) \mu_{c}}\right)}{\left(e^{n \mu_{c}}-e^{-n \mu_{c}}\right)^{7}}-\frac{1}{4}\left(N_{c}+2\right)^{2}\left(N_{c}+3\right)^{2} \frac{\left(e^{n\left(N_{c}+3\right) \mu_{c}}-e^{-n\left(N_{c}+3\right) \mu_{c}}\right)}{\left(e^{n \mu_{c}}-e^{-n \mu_{c}}\right)^{5}} .
\end{aligned}
$$

\section{B. The Phase Quenched Partition Function}

To calculate the average phase factor according to the definition (2) for $N_{f}=2$, we also need the one-dimensional phase quenched QCD partition function for two flavors which will be evaluated in this subsection. It is defined by

$$
\begin{aligned}
Z_{1+1^{*}}\left(\mu_{c}, \mu\right) & =\int_{U \in U\left(N_{c}\right)} d U \operatorname{det} D \operatorname{det} D^{\dagger} \\
& =e^{2 \mu n N_{c}} \int_{U \in U\left(N_{c}\right)} d U \operatorname{det}\left(1-e^{n\left(\mu-\mu_{c}\right)} U\right) \operatorname{det}\left(1-e^{n\left(-\mu-\mu_{c}\right)} U^{\dagger}\right) \operatorname{det}\left(1-e^{n\left(\mu-\mu_{c}\right)} U^{\dagger}\right) \operatorname{det}\left(1-e^{n\left(-\mu-\mu_{c}\right)} U\right) .
\end{aligned}
$$

This partition function is easily evaluated using the integration formulae of [15]. We find

$$
\begin{aligned}
Z_{1+1^{*}}\left(\mu_{c}, \mu\right)= & \frac{\cosh \left(2 n\left(N_{c}+2\right) \mu_{c}\right)}{8 \sinh \left(n\left(\mu_{c}-\mu\right)\right) \sinh \left(n\left(\mu+\mu_{c}\right)\right) \sinh ^{2}\left(n \mu_{c}\right)}+\frac{\left.\cosh \left(2 n\left(N_{c}+2\right) \mu\right)\right)}{8 \sinh \left(n\left(\mu-\mu_{c}\right)\right) \sinh \left(n\left(\mu+\mu_{c}\right)\right) \sinh ^{2}(n \mu)} \\
& +\frac{1}{8 \sinh ^{2}(n \mu) \sinh ^{2}\left(n \mu_{c}\right)} .
\end{aligned}
$$

For $\mu=0$ this result agrees with the $N_{f}=2$ partition function given in (28). Its microscopic limit simplifies to

$$
Z_{1+1^{*}}^{\text {micro }}\left(\mu_{c}, \mu\right)=\frac{e^{2 n N_{c} \mu_{c}}+e^{-2 n N_{c} \mu_{c}}}{16 n^{4}\left(\mu_{c}^{2}-\mu^{2}\right) \mu_{c}^{2}}+\frac{2}{16 n^{4} \mu^{2} \mu_{c}^{2}}-\frac{e^{2 n N_{c} \mu}+e^{-2 n N_{c} \mu}}{16 n^{4}\left(\mu_{c}^{2}-\mu^{2}\right) \mu^{2}} .
$$

In section VII we will show that this result is equal to the microscopic limit of the $\mathrm{QCD}_{3}$ partition function with masses $-\left(\mu_{c}+\mu\right),-\left(\mu_{c}-\mu\right), \mu_{c}-\mu, \mu_{c}+\mu$.

The large $N_{c}$ limit of the phase quenched partition function is given by

$$
\begin{aligned}
& Z_{1+1^{*}}\left(\mu_{c}, \mu\right)=\frac{e^{2 n\left(N_{c}+2\right) \mu_{c}}}{16 \sinh \left(n\left(\mu_{c}-\mu\right)\right) \sinh \left(n\left(\mu+\mu_{c}\right)\right)\left(\sinh \left(n \mu_{c}\right)\right)^{2}} \quad \text { for } \quad \mu<\mu_{c} \\
& Z_{1+1^{*}}\left(\mu_{c}, \mu\right)=\frac{e^{2 n\left(N_{c}+2\right) \mu}}{16 \sinh \left(n\left(\mu-\mu_{c}\right)\right) \sinh \left(n\left(\mu+\mu_{c}\right)\right)(\sinh (n \mu))^{2}} \quad \text { for } \quad \mu>\mu_{c} \\
& Z_{1+1^{*}}\left(\mu_{c}, \mu\right)=\frac{N_{c} e^{2 n\left(N_{c}+2\right) \mu_{c}}}{8 \sinh ^{2}\left(n \mu_{c}\right) \sinh \left(2 n \mu_{c}\right)}\left[1+O\left(N_{c}^{-1}\right)\right] \quad \text { for } \quad \mu=\mu_{c}
\end{aligned}
$$

which will be used to calculate the phase quenched average phase factor in this limit.

\section{AVERAGE PHASE FACTOR FOR $U\left(N_{c}\right)$}

In this section we will evaluate the average phase factor, first from the ratio of the full QCD partition function and the phase quenched partition function and in the next subsection starting from the definition in Eq. (3). 


\section{A. Phase Quenched Average Phase Factor}

For $N_{f}=2$, the average phase factor with the absolute value of the fermion determinant as weight is given by

$$
\left\langle e^{2 i \theta}\right\rangle_{\mathrm{pq}}=\frac{\left\langle e^{2 i \theta}|\operatorname{det}(D)|^{2}\right\rangle}{\left\langle|\operatorname{det}(D)|^{2}\right\rangle}=\frac{Z_{N_{f}=2}\left(\mu_{c}, \mu\right)}{Z_{1+1^{*}}\left(\mu_{c}, \mu\right)} .
$$

The two-flavor partition function was evaluated in subsection III A, and the phase quenched partition function was calculated in subsection III B. The large $N_{c}$ limit of the phase quenched average phase factor is given by

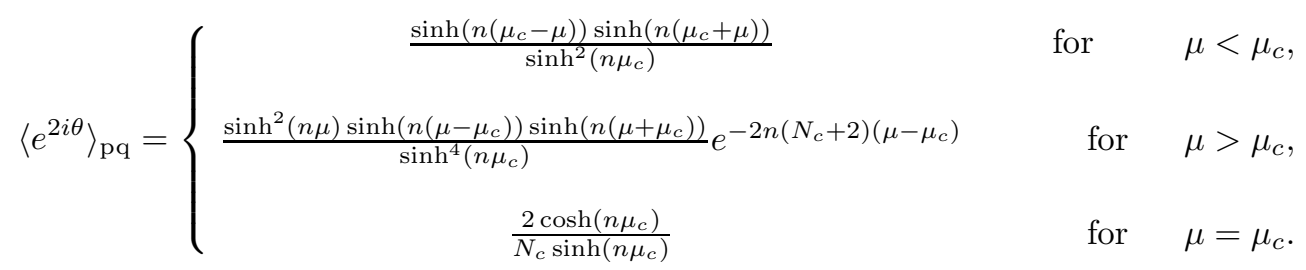

The large $\mu_{c} N_{c}, \mu N_{c}$ limit of the microscopic phase quenched partition function (34) for $\mu<\mu_{c}$ is given by

$$
Z_{\mathrm{pq}}\left(\mu_{c}, \mu\right)=\frac{e^{2 n N_{c} \mu_{c}}}{16 n^{4}\left(\mu_{c}^{2}-\mu^{2}\right) \mu_{c}^{2}}
$$

whereas the two-flavor partition function in this limit reads

$$
Z_{N_{f}=2}\left(\mu_{c}, \mu\right)=\frac{e^{2 n N_{c} \mu_{c}}}{16 n^{4} \mu_{c}^{4}}
$$

so that the average phase factor simplifies to

$$
\left\langle e^{2 i \theta}\right\rangle_{\mathrm{pq}}=1-\frac{\mu^{2}}{\mu_{c}^{2}} \quad \text { for } \quad \mu<\mu_{c} .
$$

This result is in agreement with the mean field result (16). Indeed, the denominator of (38) is the product of the Goldstone masses $\mu_{c}-\mu, \mu_{c}+\mu, \mu_{c}$ and $\mu_{c}$, whereas the denominator of (40) is the product of four Goldstone masses $\mu_{c}$. The free energy of both partition functions is equal to $2 n N_{c} \mu_{c}$.

For $\mu>\mu_{c}$, the large $N_{c}$ limit of the two flavor partition function is still given by (39), but the large $N_{c} \mu$ limit of the microscopic phase quenched partition function is now given by

$$
Z_{\mathrm{pq}}=\frac{e^{2 n N_{c} \mu}}{16 n^{4}\left(\mu^{2}-\mu_{c}^{2}\right) \mu^{2}}
$$

resulting in the average phase factor

$$
\left\langle e^{2 i \theta}\right\rangle_{\mathrm{pq}}=\frac{Z_{N_{f}=2}}{Z_{\mathrm{pq}}}=\frac{\mu^{2}}{\mu_{c}^{2}}\left(\frac{\mu^{2}}{\mu_{c}^{2}}-1\right) e^{-2 n N_{c}\left(\mu-\mu_{c}\right)} \quad \text { for } \quad \mu>\mu_{c} .
$$

This result can also be obtained from the small $n \mu, n \mu_{c}$ expansion of the large $N_{c}$ limit of the average phase factor (see Eq. (37)) at fixed $n N_{c} \mu_{c}$. We conclude that the sign problem becomes exponentially hard for $\mu>\mu_{c}$.

In Fig. 1 we show the average phase factor for different values of $n$ and $N_{c}$ and a critical chemical potential of $\mu_{c}=0.1$. In the thermodynamic limit at fixed $N_{c}$, the average phase factor is one for $\mu<\mu_{c}$ and jumps to zero for $\mu>\mu_{c}$. In the right figure we observe a rapid convergence to the mean field result discussed in section $\amalg$.

\section{B. Average phase factor for full QCD}

The average phase factor with the $N_{f}$-flavor quark determinant as weight is defined by

$$
\begin{aligned}
\left\langle e^{2 i \theta}\right\rangle_{N_{f}} & =\frac{1}{Z_{N_{f}}\left(\mu_{c}, \mu\right)} \int d U \frac{\operatorname{det} D}{\operatorname{det} D^{\dagger}} \operatorname{det}^{N_{f}} D \\
& =\frac{e^{n N_{f} N_{c} \mu_{c}}}{Z_{N_{f}}\left(\mu_{c}, \mu\right)} \int d U \frac{\operatorname{det}\left(1-U e^{n \mu-n \mu_{c}}\right) \operatorname{det}\left(1-U^{\dagger} e^{-n \mu-n \mu_{c}}\right)}{\operatorname{det}\left(1-U e^{-n \mu-n \mu_{c}}\right) \operatorname{det}\left(1-U^{\dagger} e^{n \mu-n \mu_{c}}\right)} \operatorname{det}^{N_{f}}\left(1-U e^{n \mu-n \mu_{c}}\right) \operatorname{det}^{N_{f}}\left(1-U^{\dagger} e^{-n \mu-n \mu_{c}}\right) .
\end{aligned}
$$



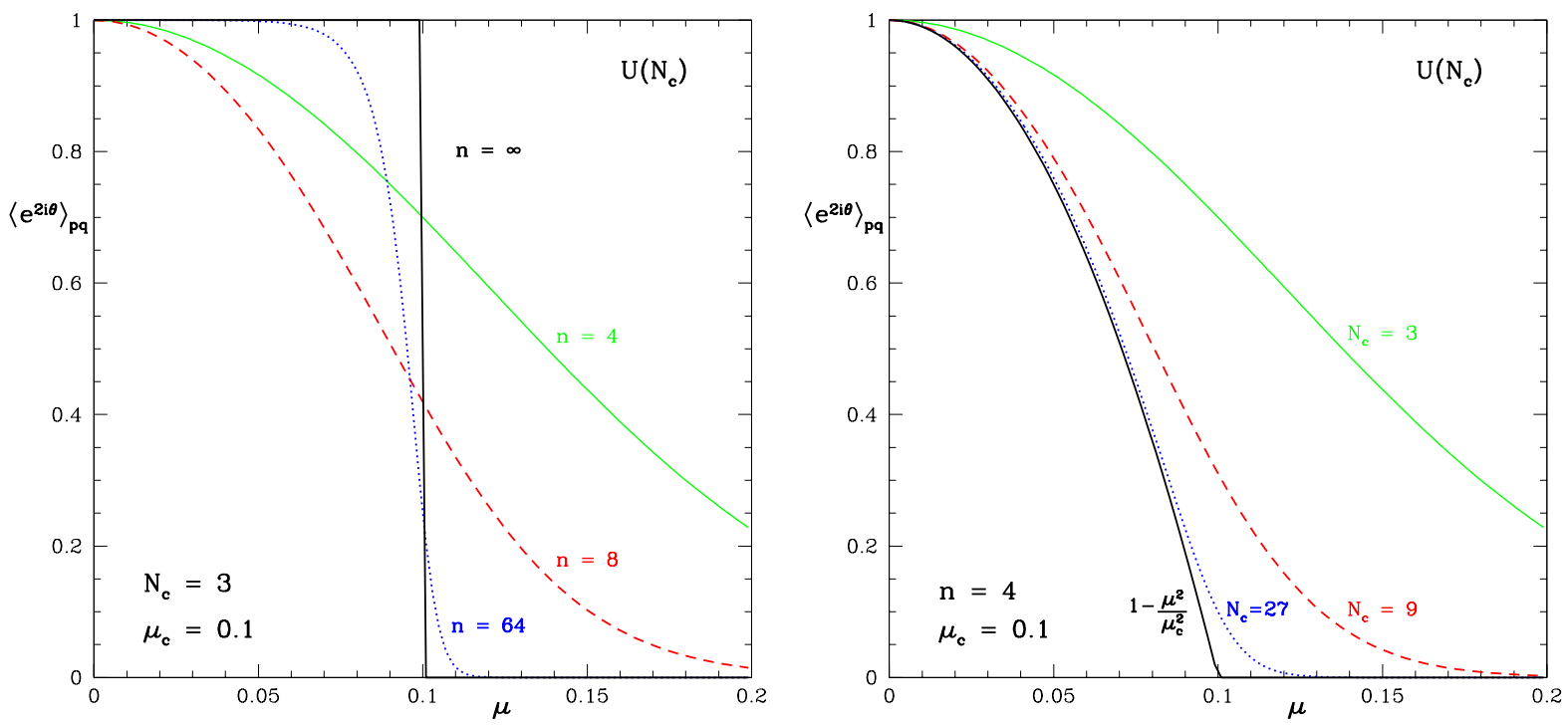

FIG. 1: The average phase factor for one-dimensional QCD with gauge group $U\left(N_{c}\right)$ calculated as the ratio of the two flavor flavor partition function and the phase quenched partition function. In the left figure the number of lattice points varies as indicated for fixed $N_{c}=3$, and in the right figure the number of colors varies as indicated for fixed $n=4$.

We evaluate this integral exactly for $N_{f}=0$ and $N_{f}=1$ but only give its large $N_{c}$ limit for other values of $N_{f}$.

Using the CFZ-formula (18) [15] we obtain in the quenched case

$$
\left\langle e^{2 i \theta}\right\rangle_{N_{f}=0}=\frac{\left(1-e^{-2 n\left(\mu+\mu_{c}\right)}\right)\left(1-e^{2 n\left(\mu-\mu_{c}\right)}\right)}{\left(1-e^{-2 n \mu_{c}}\right)^{2}}+e^{-2 n N_{c} \mu_{c}} \frac{\left(1-e^{-2 n \mu}\right)\left(1-e^{2 n \mu}\right)}{\left(1-e^{2 n \mu_{c}}\right)\left(1-e^{-2 n \mu_{c}}\right)} \quad \text { for } \quad \mu<\mu_{c} .
$$

The large $N_{c}$ limit of this result coincides with the large $N_{c}$ limit of the phase quenched average phase factor. In the microscopic limit where $\mu_{c} n N_{c}$ and $\mu n N_{c}$ remain fixed for $N_{c} \rightarrow \infty$ we obtain

$$
\left\langle e^{2 i \theta}\right\rangle_{N_{f}=0}=1-\frac{\mu^{2}}{\mu_{c}^{2}}-\frac{\mu^{2}}{\mu_{c}^{2}} e^{-2 n N_{c} \mu_{c}} .
$$

In the large $n N_{c} \mu_{c}$ limit the second term does not contribute resulting in

$$
\left\langle e^{2 i \theta}\right\rangle_{N_{f}=0}=1-\frac{\mu^{2}}{\mu_{c}^{2}},
$$

in agreement with the results obtained in [4] (see subsection[IC). In the thermodynamic limit at fixed $N_{c}$ the average phase factor converges to one for $\mu<\mu_{c}$.

After rewriting the determinants in (43) as

$$
\frac{\operatorname{det}\left(1-U^{\dagger} e^{-n \mu-n \mu_{c}}\right)}{\operatorname{det}\left(1-U^{\dagger} e^{n \mu-n \mu_{c}}\right)} \rightarrow e^{-2 n N_{c} \mu} \frac{\operatorname{det}\left(1-U e^{n \mu+n \mu_{c}}\right)}{\operatorname{det}\left(1-U e^{-n \mu+n \mu_{c}}\right)}
$$

the denominator of (43) can be expanded in powers of $U$ for $\mu>\mu_{c}$. For $N_{f}=0$ only the constant term in the integrand yields a non-vanishing result. We thus find

$$
\left\langle e^{2 i \theta}\right\rangle_{N_{f}=0}=e^{-2 n N_{c} \mu} \quad \text { for } \quad \mu>\mu_{c}
$$

so that the average phase factor vanishes in the large $n N_{c} \mu$ limit.

In Fig. 2 we show the quenched average phase factor for various values of $n$ and $N_{c}$. Also in this case the average phase factor jumps from 1 to 0 at $\mu=\mu_{c}$ in the thermodynamic limit. The convergence to the mean field result for increasing $N_{c}$ (right figure) is very rapid. This can be understood from the expansion of the microscopic result given by $1-\left(\mu^{2} / \mu_{c}^{2}\right)\left(1-\frac{1}{3}(n \mu)^{2}+\cdots\right.$. 

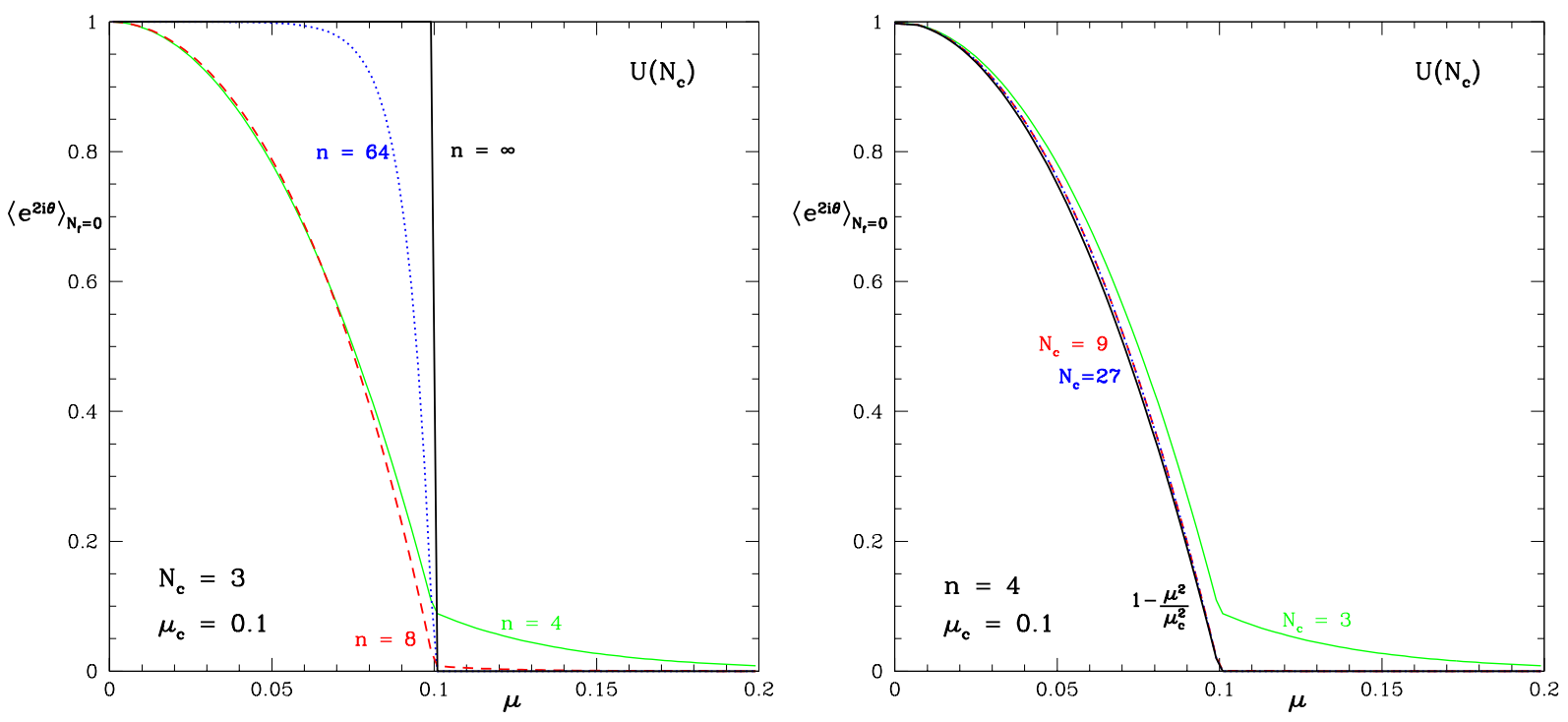

FIG. 2: The quenched average phase factor for one-dimensional QCD with gauge group $U\left(N_{c}\right)$. In the left figure $N_{c}=3$ and the number of lattice points is as indicated. In the right figure the number of colors varies whereas $n=4$.

Finally we calculate the average phase factor for an arbitrary number of flavors in the large $N_{c}$ limit. The CFZ formula can again be applied after inserting the factor

$$
\operatorname{det}^{N_{f}}\left(1-U e^{-\alpha}\right) \operatorname{det}^{N_{f}}\left(1-U^{\dagger} e^{-\beta}\right)
$$

in the denominator and taking the limit $\alpha \rightarrow \infty$ and $\beta \rightarrow \infty$ at the end of the calculation.

For $\mu<\mu_{c}$, the leading order large $N_{c}$ result is given by the identity permutation in the sum over permutations in the CFZ formula. This results in

$$
\left\langle e^{2 i \theta}\right\rangle_{N_{f}}=\left(\frac{\left(1-e^{-2 n\left(\mu+\mu_{c}\right)}\right)\left(1-e^{2 n\left(\mu-\mu_{c}\right)}\right)}{\left(1-e^{-2 n \mu_{c}}\right)^{2}}\right)^{N_{f}+1} \quad \text { for } \quad \mu<\mu_{c} .
$$

The microscopic limit of this result is given by

$$
\left\langle e^{2 i \theta}\right\rangle_{N_{f}}=\left[1-\frac{\mu^{2}}{\mu_{c}^{2}}\right]^{N_{f}+1} \quad \text { for } \quad \mu<\mu_{c}
$$

in agreement with the discussion given in section IIC

For $\mu>\mu_{c}$ the CFZ formula can again be applied after rewriting the partition function according to (46) and the insertion (48). Because of degenerate prefactors, all critical chemical potentials that occur in the combination $\mu+\mu_{c}$ have to be taken different. After carefully taking limits we find

$$
Z_{N_{f}+1 \mid 1^{*}}\left(\mu_{c}, \mu\right)=\frac{N_{c}^{N_{f}}}{N_{f} !} e^{-2 \mu n N_{c}} \frac{e^{\mu_{c} n N_{c} N_{f}}}{\left(1-e^{-2 \mu_{c} n}\right)^{N_{f}^{2}}} \frac{\left(1-e^{-2 n\left(\mu+\mu_{c}\right)}\right)^{N_{f}}\left(1-e^{-2 n \mu}\right)^{N_{f}}}{\left(1-e^{-2 n \mu_{c}}\right)^{N_{f}}} .
$$

The average phase factor given by

$$
\left\langle e^{2 i \theta}\right\rangle_{N_{f}}=\frac{Z_{N_{f}+1 \mid 1^{*}}}{Z_{N_{f}}} \sim e^{-2 n N_{c} \mu} \quad \text { for } \quad \mu>\mu_{c}
$$

vanishes in the thermodynamic limit. The exact result becomes rather cumbersome even for small values of $N_{f}$. As an illustration we give the result for $Z_{2 \mid 1^{*}}\left(\mu_{c}, \mu\right)$ in Appendix A . 


\section{AVERAGE PHASE FACTOR FOR $S U\left(N_{c}\right)$}

To calculate integrals over $S U\left(N_{c}\right)$ we use the identity

$$
\int_{S U\left(N_{c}\right)} d U \cdots=\sum_{p=-\infty}^{\infty} \int_{U\left(N_{c}\right)} d U \operatorname{det}^{p}(U) \cdots
$$

For the partition functions discussed below, with a few exceptions the sum truncates to a small number of terms.

\section{A. $S U\left(N_{c}\right)$ QCD Partition Function}

Let us first calculate the $1 d S U\left(N_{c}\right)$ QCD partition function for $N_{f}=1$. The partition function is defined by

$Z_{N_{f}}^{S U\left(N_{c}\right)}\left(\mu_{c}, \mu\right)=\int_{S U\left(N_{c}\right)} d U \operatorname{det}^{N_{f}} D=\sum_{p=-\infty}^{\infty} \int_{U\left(N_{c}\right)} d U \operatorname{det}^{p} U e^{n N_{c} \mu_{c}} \operatorname{det}^{N_{f}}\left(1+U e^{n \mu-n \mu_{c}}\right) \operatorname{det}^{N_{f}}\left(1+U^{\dagger} e^{-n \mu-n \mu_{c}}\right)$

For $p \geq N_{f}$ the integrand can be rewritten in terms of determinant of matrices $U$ only

$$
\begin{aligned}
Z_{N_{f}, p}\left(\mu_{c}, \mu\right) & =\int_{U\left(N_{c}\right)} d U \operatorname{det}^{p} U \operatorname{det}^{N_{f}} D \\
& =\int_{U\left(N_{c}\right)} d U e^{n N_{c} N_{f} \mu_{c}} \operatorname{det}^{p-N_{f}}(U) \operatorname{det}^{N_{f}}\left(1+U e^{n \mu-n \mu_{c}}\right) \operatorname{det}^{N_{f}}\left(U+e^{-n \mu-n \mu_{c}}\right) .
\end{aligned}
$$

For $p>N_{f}$ it follows immediately that the integral vanishes. For $p=N_{f}$ only the constant term inside the determinants contributes to integral resulting in

$$
\begin{aligned}
& Z_{N_{f}, p=N_{f}}\left(\mu_{c}, \mu\right)=e^{-n N_{f} N_{c} \mu}, \\
& Z_{N_{f}, p>N_{f}}\left(\mu_{c}, \mu\right)=0 .
\end{aligned}
$$

For $p<0$ we combine $\operatorname{det}^{p} U=\operatorname{det}^{-p} U^{\dagger}$ with the factor $\operatorname{det}^{N_{f}}\left(1-U e^{n \mu-n \mu_{c}}\right)$. We then find

$$
\begin{aligned}
& Z_{N_{f}, p=-N_{f}}\left(\mu_{c}, \mu\right)=e^{n N_{f} N_{c} \mu}, \\
& Z_{N_{f}, p<-N_{f}}\left(\mu_{c}, \mu\right)=0 .
\end{aligned}
$$

Using that the hermitean conjugate of a unitary matrix is also unitary we obtain

$$
Z_{N_{f},-p}\left(\mu_{c}, \mu\right)=Z_{N_{f}, p}\left(\mu_{c},-\mu\right)
$$

For $|p|<N_{f}$ the integral can be calculated by means of the CFZ formula by choosing all $\mu_{c}$ different and introducing the limit

$$
\operatorname{det} U=\lim _{\alpha \rightarrow \infty} \operatorname{det}\left(U-e^{-\alpha}\right) \text {. }
$$

The limit of degenerate $\mu_{c}$ and $\alpha \rightarrow \infty$ is taken at the end of the calculation. We will only give exact results for $N_{f}=1$ and $N_{f}=2$.

Using (55|56) and the result for $U\left(N_{c}\right)$ we obtain for $N_{f}=1$

$$
Z_{N_{f}=1}^{S U\left(N_{c}\right)}\left(\mu_{c}, \mu\right)=e^{-n N_{f} N_{f} \mu}+e^{n N_{f} N_{f} \mu}+\frac{\sinh \left(n\left(N_{c}+1\right) \mu_{c}\right)}{\sinh \left(n \mu_{c}\right)}
$$

in agreement with earlier work by Bilic and Demeterfi [24].

For $N_{f}=2$, the only integrals that have not yet been calculated are those for $p= \pm 1$ which are related by (57). For the $p=1$ contribution we find

$$
Z_{N_{f}=2, p=1}\left(\mu_{c}, \mu\right)=e^{n N_{c}\left(\mu_{c}-\mu\right)}\left[N_{c} \frac{e^{-2 n\left(N_{c}+1\right) \mu_{c}}+e^{2 n \mu_{c}}}{\left(e^{n \mu_{c}}-e^{-n \mu_{c}}\right)^{2}}-\frac{e^{-2 n N_{c} \mu_{c}}\left(e^{-3 n \mu_{c}}-3 e^{-n \mu_{c}}\right)-e^{3 n \mu_{c}}+3 e^{n \mu_{c}}}{\left(e^{n \mu_{c}}-e^{-n \mu_{c}}\right)^{3}}\right] .
$$


For $\mu<\mu_{c}$ the large $n N_{c} \mu$ limit of the $N_{f}=2$ partition function is dominated by the $p=0$ term, whereas for $\mu>\mu_{c}$ the large $n N_{c} \mu_{c}$ limit is given by the $p=-2$ term. For $\mu=\mu_{c}$ the large $N_{c}$ limit is dominated by the $p=-1$ with $1 / N_{c}$ corrections from the $p=0$ and $p=-2$ terms. We thus find as leading large $N_{c}$ result

$$
\begin{aligned}
& Z_{N_{f}=2}^{S U\left(N_{c}\right)}\left(\mu_{c}, \mu\right)=\frac{e^{2 n\left(N_{c}+2\right) \mu_{c}}}{16 \sinh ^{4}\left(n \mu_{c}\right)} \text { for } \mu<\mu_{c}, \\
& Z_{N_{f}=2}^{S U\left(N_{c}\right)}\left(\mu_{c}, \mu\right)=e^{2 n N_{c} \mu} \quad \text { for } \mu>\mu_{c} \\
& Z_{N_{f}=2}^{S U\left(N_{c}\right)}\left(\mu_{c}, \mu\right)=\frac{N_{c} e^{2 n\left(N_{c}+1\right) \mu_{c}}}{4 \sinh ^{2}\left(n \mu_{c}\right)}\left[1+O\left(N_{c}^{-1}\right)\right] \quad \text { for } \mu=\mu_{c} .
\end{aligned}
$$

Both for $\mu<\mu_{c}$ and $\mu>\mu_{c}$ the terms that are canceled by the integration over the unitary group are subleading in the thermodynamic limit. In other words there is no serious sign problem.

By inspection one can easily show that the dominance of the $p=0$ terms for $\mu<\mu_{c}$ and the $p=-N_{f}$ term for $\mu>\mu_{c}$ is a feature of the large $N_{c}$ limit that is valid for any number of flavors. Therefore, for $\mu<\mu_{c}$, the chiral condensate is the same as for the $U\left(N_{c}\right)$ theory. For $\mu>\mu_{c}$ though, the dominant $p=-N_{f}$ term is mass-independent. This results in a vanishing chiral condensate so that the large $N_{c}$ limit of the $U\left(N_{c}\right)$ theory and the $S U\left(N_{c}\right)$ theory is different.

\section{B. The Phase Quenched Partition Function for $S U\left(N_{c}\right)$}

A second ingredient for the average phase factor is the phase quenched $S U\left(N_{c}\right)$ partition function. Using the same arguments as for the full QCD partition function, one easily obtains

$$
\begin{aligned}
Z_{1+1^{*},|p|>2}\left(\mu_{c}, \mu\right) & =0 \\
Z_{1+1^{*},|p|=2}\left(\mu_{c}, \mu\right) & =1, \\
Z_{1+1^{*},-p}\left(\mu_{c}, \mu\right) & =Z_{1+1^{*}, p}\left(\mu_{c},-\mu\right) .
\end{aligned}
$$

The partition function for $p=0$ is the $U\left(N_{c}\right)$ partition function which was already given in (33). What remains to be calculated is the partition function for $p=+1$. Using the CFZ formula one easily arrives at

$$
Z_{1+1^{*}, p=1}\left(\mu_{c}, \mu\right)=\frac{1}{4 \sinh (n \mu) \sinh \left(n \mu_{c}\right)}\left(\frac{\sinh \left(n\left(N_{c}+2\right)\left(\mu+\mu_{c}\right)\right)}{\sinh \left(n\left(\mu+\mu_{c}\right)\right)}-\frac{\sinh \left(n\left(N_{c}+2\right)\left(\mu-\mu_{c}\right)\right)}{\left.\sinh \left(n\left(\mu-\mu_{c}\right)\right)\right)}\right) .
$$

Both for $\mu<\mu_{c}$ and $\mu>\mu_{c}$, the leading large $N_{c}$ result of the phase quenched partition function resides in the $p=0$ term given in Eq. (35).

\section{Average Phase Factor for $S U\left(N_{c}\right)$}

The average phase factor can be either obtained from the ratio of the full QCD partition function and the phase quenched partition function or can be calculated with the full QCD partition function as weight (see Eqs. (2) and (3)). In the first case we find for large $N_{c}$,

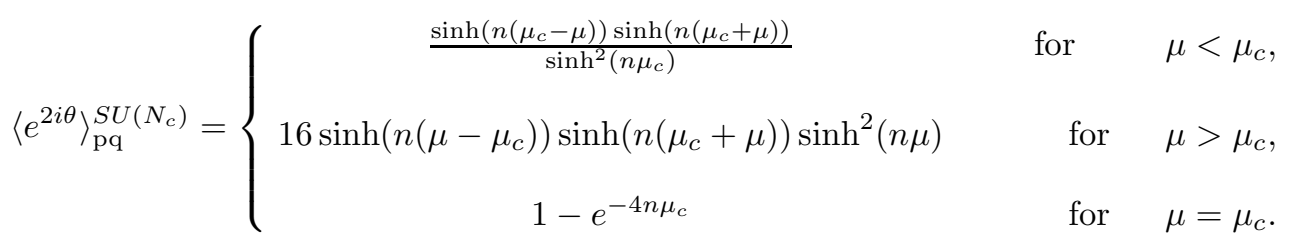

The microscopic limit of the average phase factor is obtained by expanding this result for small $\mu_{c}, \mu$. We again find the usual mean field result

$$
\left\langle e^{2 i \theta}\right\rangle_{\mathrm{pq}}^{S U\left(N_{c}\right)}=\left(1-\frac{\mu^{2}}{\mu_{c}^{2}}\right) \theta\left(\mu_{c}-\mu\right)
$$

In the thermodynamic limit, the average phase factor converges to one in both for $\mu<\mu_{c}$ and $\mu>\mu_{c}$. 

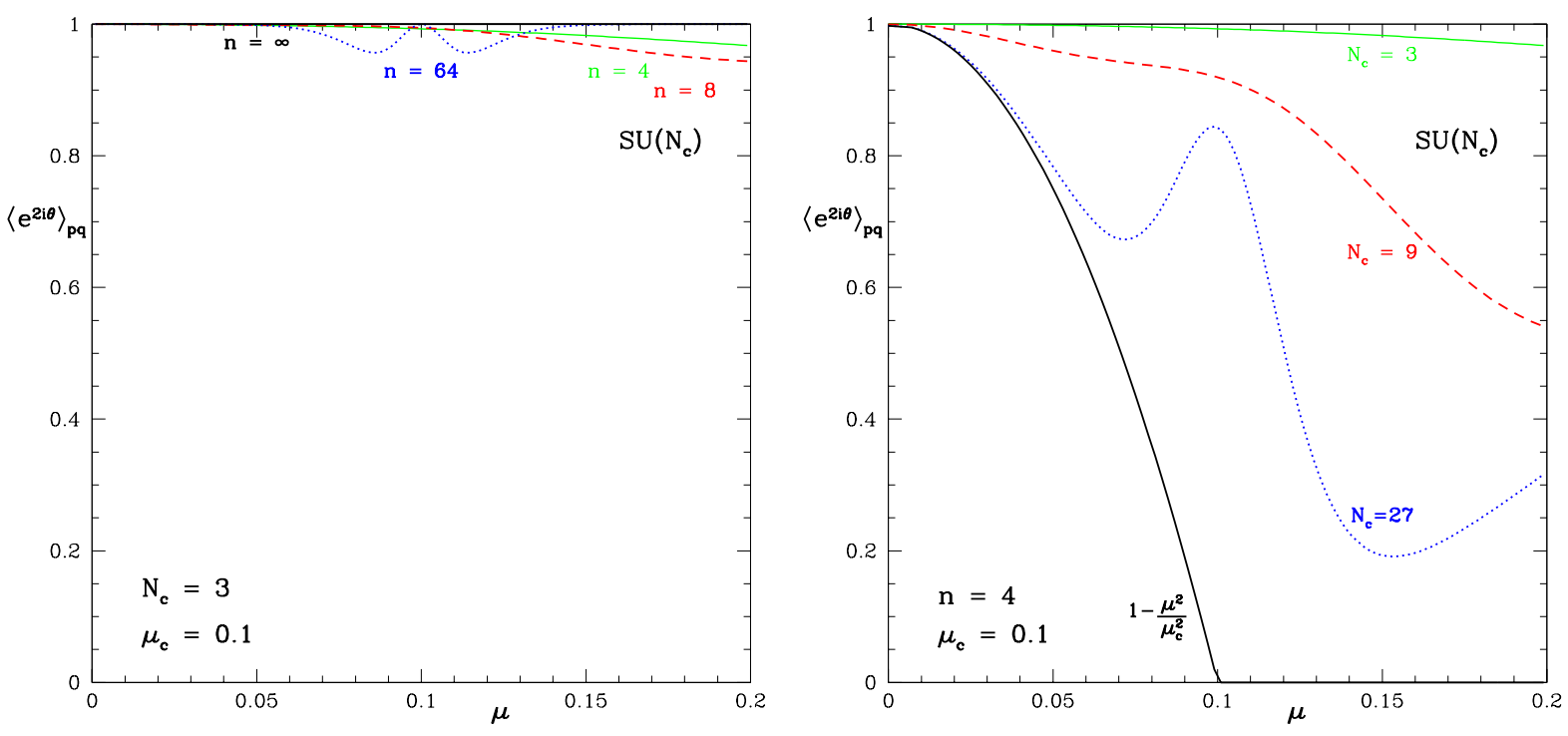

FIG. 3: The phase quenched average phase factor for one-dimensional QCD with gauge group $S U\left(N_{c}\right)$. In the left figure $N_{c}=3$ and the number of lattice points is as indicated. In the right figure the number of colors varies whereas $n=4$.

Exact results for the average phase factor are displayed in Fig. 3. At finite $N_{c}$, we observe a rapid convergence to the asymptotic value of 1 . At fixed $n$, the approach to the large $N_{c}$ limit is slow. Also clearly visible is that for $\mu \approx \mu_{c}$ the corrections to the microscopic limit are large. As was already discussed before, this is due to the $p=-1$ contribution.

In the quenched case the average phase factor for $S U\left(N_{c}\right)$ defined according to Eq. (3) can be written as

$$
\left\langle e^{2 i \theta}\right\rangle_{N_{f}=0}^{S U\left(N_{c}\right)}=\sum_{p=-\infty}^{\infty} \int_{U \in U\left(N_{c}\right)} d U \operatorname{det}^{p}(U) \frac{\operatorname{det}\left(1+U e^{n \mu-n \mu_{c}}\right) \operatorname{det}\left(1+U^{\dagger} e^{-n \mu-n \mu_{c}}\right)}{\operatorname{det}\left(1+U e^{-n \mu-n \mu_{c}}\right) \operatorname{det}\left(1+U^{\dagger} e^{n \mu-n \mu_{c}}\right)} .
$$

The integrals can again be calculated by means of the CFZ formula. For $N_{c} \geq 3$ one can easily show that the limit introduced in (58) gives vanishing results for $|p|>2$. However, for $N_{c}=1$ and $N_{c}=2$ additional terms may contribute to this limit. For $N_{c}=2$, where the average phase factor is equal to one, contributions for $|p|>2$ vanish for $\mu<\mu_{c}$, but terms with $p<-2$ are nonzero for $\mu>\mu_{c}$. The formulae for $|p| \leq 2$ given below are valid for $N_{c}=2$. For $N_{c}=1$ the integrals in (66) only vanish for $p \geq 2$ and $\mu>\mu_{c}$. Also the general answer for $p=-2$ is not correct in this case.

For $p=-1$ we obtain

$$
\left\langle e^{2 i \theta}\right\rangle_{N_{f}=0}=\left\{\begin{array}{cc}
e^{-N_{c} n\left(\mu_{c}-\mu\right)} \frac{\left(1-e^{-2 n\left(\mu_{c}+\mu\right)}\right)\left(1-e^{-2 n \mu}\right)}{1-e^{-2 n \mu_{c}}} & \text { for } \quad \mu<\mu_{c}, \\
\frac{e^{n N_{c}\left(\mu_{c}-\mu\right)}\left(1-e^{-2 n \mu}\right)}{1-e^{-2 n \mu_{c}}}\left[\left(1-e^{-2 n \mu_{c}-2 n \mu}\right)-e^{-2 n N_{c} \mu_{c}}\left(e^{-2 n \mu_{c}}-e^{-2 n \mu}\right)\right] & \text { for } \quad \mu>\mu_{c} .
\end{array}\right.
$$

For $p=1$ we find

$$
\left\langle e^{2 i \theta}\right\rangle_{N_{f}=0}=\left\{\begin{array}{ccc}
e^{-N_{c} n\left(\mu_{c}+\mu\right) \frac{\left(1-e^{-2 n\left(\mu_{c}-\mu\right)}\right)\left(1-e^{2 n \mu}\right)}{1-e^{-2 n \mu_{c}}}} & \text { for } & \mu<\mu_{c} \\
0 & \text { for } & \mu>\mu_{c} .
\end{array}\right.
$$

Finally, for $p=-2$ the result is

$$
\left\langle e^{2 i \theta}\right\rangle_{N_{f}=0}=\left\{\begin{array}{ccc}
0 & \text { for } & \mu<\mu_{c}, \\
\left(1-e^{-2 n \mu}\right)\left(1-e^{-2 n\left(\mu+\mu_{c}\right)}\right)\left(1-e^{2 n\left(\mu_{c}-\mu\right)}\right)\left(1-e^{-2 n \mu}\right) & \text { for } & \mu>\mu_{c} .
\end{array}\right.
$$

For $p=2$ the average phase factor vanishes. Notice that the relation

$$
\left\langle e^{2 i \theta}\right\rangle_{N_{f}=0,-p}\left(\mu_{c}, \mu\right)=\left\langle e^{2 i \theta}\right\rangle_{N_{f}=0, p}\left(\mu_{c},-\mu\right)
$$



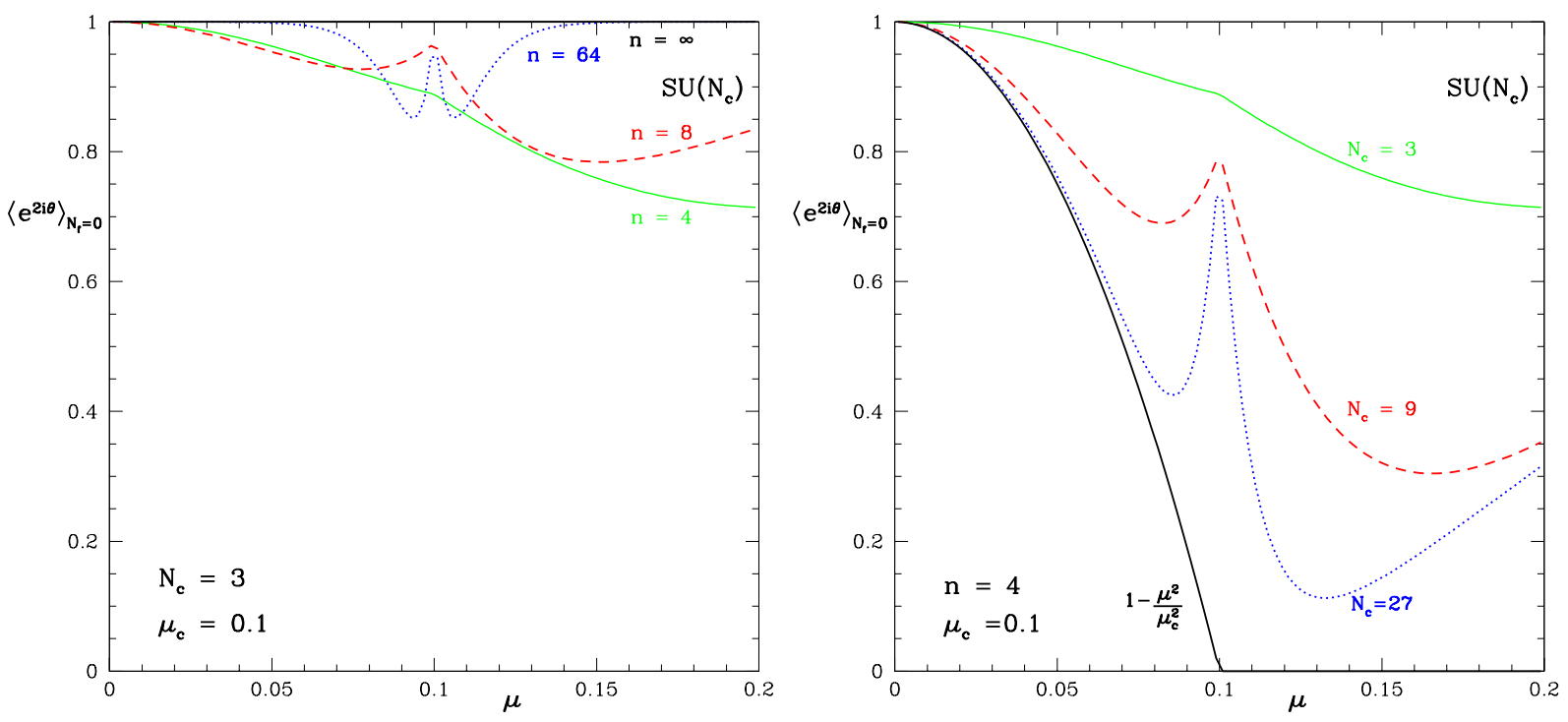

FIG. 4: Average phase factor for one-dimensional QCD with gauge group $S U\left(N_{c}\right)$. In the left figure $N_{c}=3$ and the number of lattice points is as indicated. In the right figure the number of colors varies whereas $n=4$.

is also valid in this case. It can be used to derive results for negative values of $\mu$.

In the large $N_{c}$ limit, the average phase factor is dominated by the $p=0$ contribution for $\mu<\mu_{c}$ and by the $p=-2$ contribution for $\mu>\mu_{c}$. For $\mu=\mu_{c}$, the $p=0, p=-1$ and $p=-2$ contributions are of equal order in $N_{c}$, but only the $p=-1$ term is nonvanishing. As large $N_{c}$ limit of the average phase factor we thus find

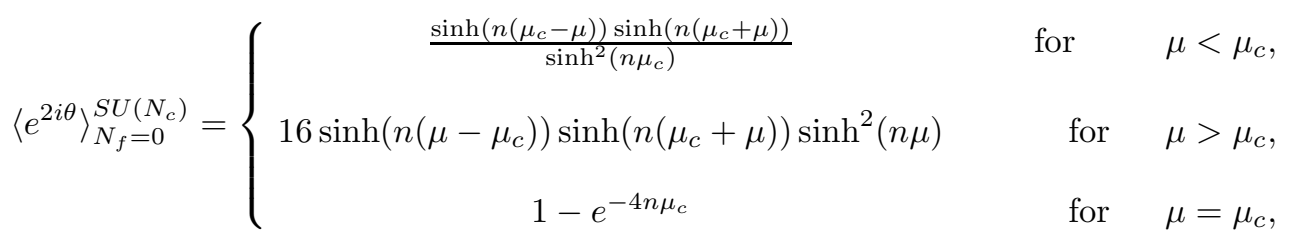

which is the same expression as obtained in (64) for the phase quenched average phase factor. The microscopic limit of the average phase factor is again given by the small $\mu-\mu_{c}$ expansion of this result which is equal to the usual mean field result

$$
\left\langle e^{2 i \theta}\right\rangle_{N_{f}=0}^{S U\left(N_{c}\right)}=\left(1-\frac{\mu^{2}}{\mu_{c}^{2}}\right) \theta\left(\mu_{c}-\mu\right)
$$

In Fig. 4 we show the exact result for the $S U\left(N_{c}\right)$ average phase factor. We observe a rapid approach to the thermodynamic limit at fixed $N_{c}$ just like in Fig. 3.

In the large $N_{c}$ limit the result for the quenched average phase calculated according to (66) is also given by (64). As already was argued in the introduction, we conclude that QCD in one dimension with $S U\left(N_{c}\right)$ as gauge group does not have a serious sign problem, not only for $\mu<\mu_{c}$, but also for $\mu>\mu_{c}$. Indeed one-dimensional QCD at nonzero chemical potential could be simulated reliably by the Glasgow method [26].

\section{THE SIGN PROBLEM AT NONZERO TEMPERATURE}

The results of the previous two sections show that the sign problem is severe for QCD in one dimension with gauge group $U\left(N_{c}\right)$ and $\mu>\mu_{c}$. Although this theory does not have a critical temperature, it is clear from Fig. 1 that the average phase factor for $\mu>\mu_{c}$ increases significantly for higher temperatures (i.e. for lower values of $n$ ). However, this is a geometric effect due to the Boltzmann factor. Recent lattice simulations [10, 11, 12, 26, 38, 39, 40, 41, 42, 43, 44, 45, 46, 47] suggest that the sign problem becomes much milder for $T$ around $T_{c}$. Because of the absence of a critical temperature, this temperature dependence of the average phase factor cannot be investigated in 

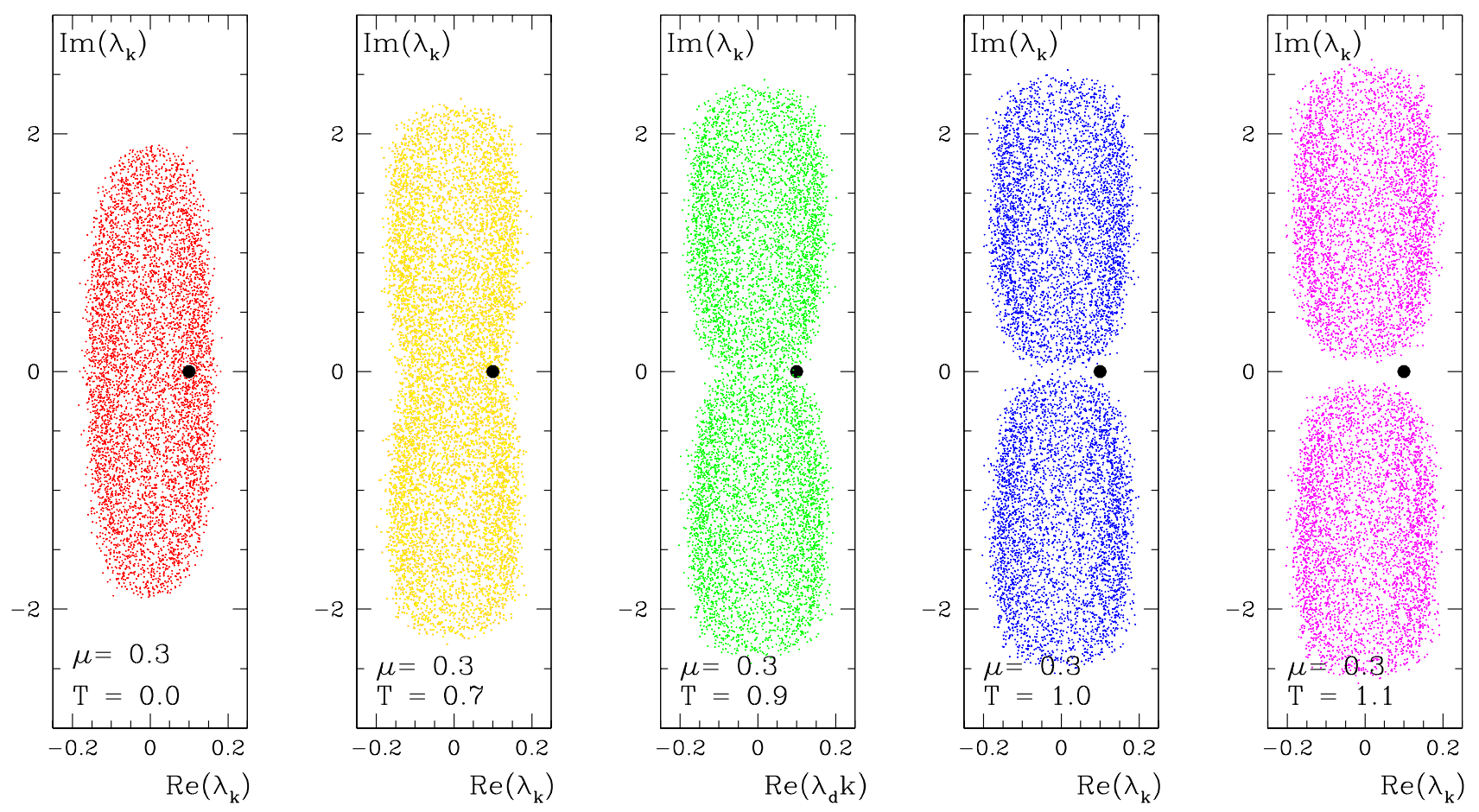

FIG. 5: Scatter plot of the eigenvalues of the random matrix Dirac operator for $\mu=0.3$ and $T=0,0.7,0.9,1.0,1.1$. The quark mass at $m=0.1$ is indicated by the black dot.

one dimensional QCD. Instead we study this problem in a schematic random matrix model at nonzero temperature and chemical potential 30, 48, 49]. This model has a severe sign problem as well as a critical temperature and its eigenvalues are scattered in a finite domain of the complex plane, just like in QCD. The quenched average phase factor in this model is given by the matrix integral

$$
\left\langle e^{2 i \theta}\right\rangle(m, \mu, T)=\int d C e^{-N \operatorname{Tr} C C^{\dagger}} \frac{\operatorname{det}(D+m)}{\operatorname{det}\left(D^{\dagger}+m\right)},
$$

with Dirac operator given by

$$
D=\left(\begin{array}{cc}
0 & i C+\mu+i t \\
i C^{\dagger}+\mu+i t & 0
\end{array}\right)
$$

and $t$ is the traceless diagonal matrix $t=\operatorname{diag}(-T, \cdots,-T, T, \cdots, T)$. The integral is over the real and imaginary parts of the matrix elements of the complex $N \times N$ matrices $C$. The phase quenched average phase factor is defined by

$$
\left\langle e^{2 i \theta}\right\rangle_{\mathrm{pq}}=\frac{\int d C \operatorname{det}^{2}(D+m) e^{-N \operatorname{Tr} C C^{\dagger}}}{\int d C \operatorname{det}(D+m) \operatorname{det}\left(D^{\dagger}+m\right) e^{-N \operatorname{Tr} C C^{\dagger}}}
$$

If at all possible, it certainly takes a significant effort to evaluate these integrals analytically. Therefore we have studied the quenched (73) and phase quenched (75) average phase factor numerically. In Fig. 5, we give scatter plots of the eigenvalues of the random matrix Dirac operator. For each of the temperatures $T=0,0.7,0.9,1,1.1$, the figures show results of 40 matrices with $N=100$ and $\mu=0.3$. The black dot represents the quark mass for which the average phase factor is calculated. In Fig. 6 we show the $\mu$-dependence of the phase quenched (left) and quenched (right) average phase factor obtained by averaging over 10,000 to 80,000 matrices $C$ with $N=100$. The results for the phase quenched average phase factor for $T=0$ and $T=0.7$ and $\mu>\mu_{c}$ are not displayed because they do not converge because of the severity of the sign problem. The average phase factor depends only weakly on the size of 

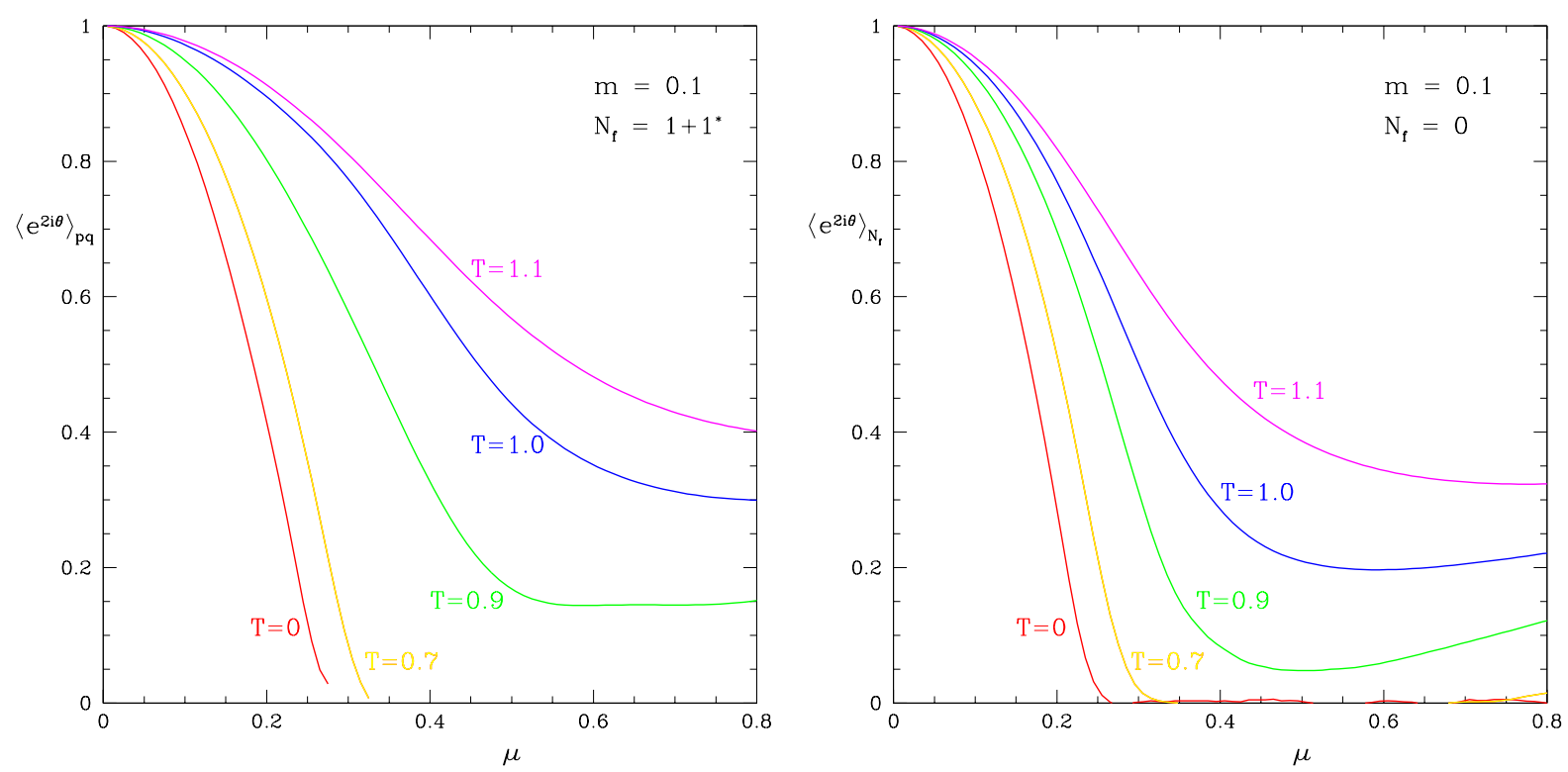

FIG. 6: Phase quenched (left) and quenched (right) average phase factor versus chemical potential. Results have been obtained from the chiral random matrix model (74).

the matrices. The conclusion of Figs. 5 and 6 is that the sign problem is not serious when the quark mass is outside the domain of the eigenvalues. This confirms the conjecture that was made in [4, 5, 50]. It also agrees with the observation in [30] that the free energy of the full theory and the phase quenched theory are the same for $T=0$ if the quark mass is outside the domain of the eigenvalues (this is the case for $\mu<\mu_{c}$ ). The $\mu$-dependence is due to the curvatures at the saddle point.

\section{COMPARISON WITH THE QCD 3 PARTITION FUNCTION}

According to the general arguments given in section II B, the microscopic limit of the one dimensional QCD partition function at nonzero chemical potential is equal to the microscopic limit of the $\mathrm{QCD}_{3}$ partition function at zero chemical potential, but with shifted quark masses. In this section we show this explicitly for some of the results derived before. We first discuss the microscopic limit of the $\mathrm{QCD}_{3}$ partition function which can be derived from either a chiral Lagrangian or a random matrix model with the same global symmetries [28]. For a discussion of $\mathrm{QCD}_{3}$ in terms of chiral Lagrangians we refer to [51].

\section{A. Random Matrix Model}

From the continuum Dirac operator (9) it follows that the random matrix model that describes the fluctuations of the low-lying Dirac eigenvalues is given by

$$
Z=\int d H P(H) \operatorname{det}(i H+\mu+m) \operatorname{det}(i H-m+\mu),
$$

where the probability distribution can conveniently be taken to be the Gaussian distribution

$$
P(H)=e^{-2 N \Sigma^{2} \operatorname{Tr} H^{2}} .
$$

Such random matrix partition functions have been studied elaborately in the literature to analyze the microscopic limit of QCD in three dimensions [28, 52, 53, 54, 55]. The microscopic limit of the partition function (76) with quark masses given by $M=\operatorname{diag}\left(-m_{1}, \cdots,-m_{N_{f}}, m_{1}, \cdots, m_{N_{f}}\right)$ is equal to [53, 54]

$$
Z_{\mathrm{QCD}_{3}}=\frac{1}{\Delta(M)} \operatorname{det}\left(\begin{array}{cc}
A(m) & A(-m) \\
A(-m) & A(m)
\end{array}\right),
$$


where the matrix elements of the $N_{f} \times N_{f}$ matrix $A(m)$ are given by

$$
A(m)_{k l}=m_{k}^{l} e^{-m_{k}},
$$

and $\Delta$ is the Vandermonde determinant

$$
\Delta(M)=\prod_{k>l}^{2 N_{f}}\left(M_{k k}-M_{l l}\right) .
$$

Although general expressions for $\mathrm{QCD}_{3}$ partition functions with an arbitrary number of bosonic and fermionic determinants are also known [56], for our purposes we only need the partition function

$$
Z_{N_{f}+2 \mid 2}=\left\langle\prod_{k=1}^{N_{f}} \operatorname{det}\left(D+m_{k}\right) \operatorname{det}\left(D-m_{k}\right) \frac{\operatorname{det}\left(D+z_{1}\right) \operatorname{det}\left(D-z_{2}\right)}{\operatorname{det}\left(D+\bar{z}_{1}\right) \operatorname{det}\left(D-\bar{z}_{2}\right)}\right\rangle .
$$

This partition function was evaluated in [57] by means of the supersymmetric method and is given by

$$
\begin{aligned}
Z_{N_{f}+2 \mid 2}= & \frac{\left(z_{1}-\bar{z}_{1}\right)\left(z_{2}-\bar{z}_{2}\right)}{\Delta_{N_{f}}^{2}(m)} \frac{\exp N_{c}\left(\bar{z}_{1}+\bar{z}_{2}\right)}{N_{c}\left(\bar{z}_{1}+\bar{z}_{2}\right)} \prod_{k=1}^{N_{f}} \frac{\left(m_{k}-\bar{z}_{1}\right)\left(m_{k}-\bar{z}_{2}\right)}{\left(m_{k}-z_{1}\right)\left(m_{k}-z_{2}\right)} \operatorname{det}\left[\frac{\frac{\sinh N_{c}\left(m_{k}+m_{l}\right)}{N_{c}\left(m_{k}+m_{l}\right)}}{\frac{\sinh N_{c}\left(z_{2}+m_{l}\right)}{N_{c}\left(z_{2}+m_{l}\right)}} \frac{\frac{\sinh N_{c}\left(m_{k}+z_{1}\right)}{N_{c}\left(m_{k}+z_{1}\right)}}{\frac{\sinh N_{c}\left(z_{1}+z_{2}\right)}{N_{c}\left(z_{1}+z_{2}\right)}}\right] \\
& +\frac{e^{-N_{c}\left(z_{1}-\bar{z}_{1}\right)+N_{c}\left(z_{2}-\bar{z}_{2}\right)}}{\Delta_{N_{f}}^{2}(m)} \operatorname{det}\left[\frac{\sinh N_{c}\left(m_{k}+m_{l}\right)}{N_{c}\left(m_{k}+m_{l}\right)}\right] .
\end{aligned}
$$

The last term is a so-called Efetov-Wegner term, and the Vandermonde determinant is over positive masses only

$$
\Delta(m)=\prod_{k>l}^{N_{f}}\left(m_{k}-m_{l}\right) .
$$

\section{B. Microscopic Limit}

We will now show that the microscopic limit of the partition function (25) is equal to the microscopic limit of the $\mathrm{QCD}_{3}$ partition function. To this end we multiply row $k$ of the first $N_{f}$ rows of the determinant by $\exp \left(-N_{c} m_{-k} / 2\right)$ and row $k$ of the second $N_{f}$ rows of the determinant in (25) by $\exp \left(-N_{c} m_{+k} / 2\right)$. In the microscopic limit we keep $N_{c} m_{ \pm, k}$ fixed so that we can expand the masses that do not occur in this combination. By subtracting successive columns starting with the first one we obtain

$$
\begin{aligned}
& Z_{N_{f}}=\frac{1}{\prod_{1 \leq k<l \leq 2 N_{f}}\left(M_{l}-M_{k}\right)}
\end{aligned}
$$

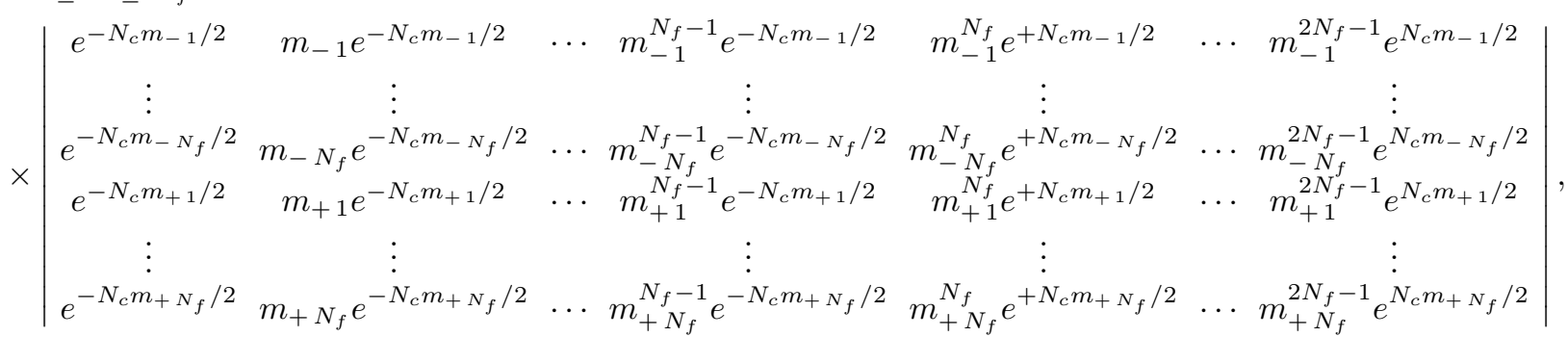

where the masses $M_{K}$ are defined below Eq. (25). By multiplying the columns $l=N_{f}+1, \cdots, 2 N_{f}$ by $(-1)^{l-1}$ and introducing microscopic masses, we obtain exactly the expression for the $\mathrm{QCD}_{3}$ partition function given in (78).

For masses $-\left(\mu_{c}+\mu\right),-\left(\mu_{c}-\mu\right), \mu_{c}-\mu, \mu_{c}+\mu$ corresponding to the microscopic limit of the two-flavor phase quenched partition function given in (34) the determinant in (84) is given by

$$
4 n \mu^{2}\left(e^{2 n N_{c} \mu_{c}}+e^{-2 n N_{c} \mu_{c}}\right)-4 n \mu_{c}^{2}\left(e^{2 n N_{c} \mu}+e^{-2 n N_{c} \mu}\right)+8 n\left(\mu_{c}^{2}-\mu^{2}\right) .
$$

and the prefactor is equal to

$$
\prod_{1 \leq k<l \leq 2 N_{f}}\left(M_{l}-M_{k}\right)=64 n^{6}\left(\mu_{c}^{2}-\mu^{2}\right) \mu_{c}^{2} \mu^{2}
$$




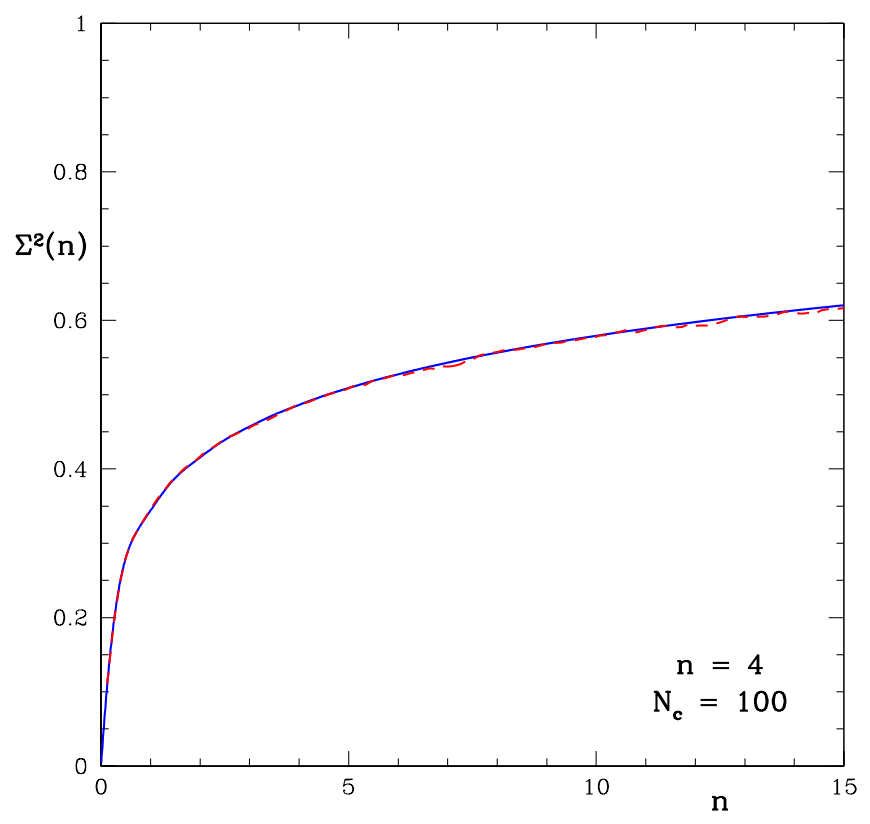

FIG. 7: The number variance for $n=4$ and $N_{c}=100$.

Their ratio coincides with the microscopic phase quenched partition function given in (34).

As second example, we consider the quenched average phase factor for $U\left(N_{c}\right)$ given in (43). For $N_{f}=0$ the partition function (82) simplifies to

$$
Z_{2 \mid 2}=\left(z_{1}-\bar{z}_{1}\right)\left(z_{2}-\bar{z}_{2}\right) \frac{e^{N_{c}\left(\bar{z}_{1}+\bar{z}_{2}\right)}}{N_{c}\left(\bar{z}_{1}+\bar{z}_{2}\right)} \frac{\sinh N_{c}\left(z_{1}+z_{2}\right)}{N_{c}\left(z_{1}+z_{2}\right)}+e^{-N_{c}\left(z_{1}-\bar{z}_{1}-z_{2}+\bar{z}_{2}\right)} .
$$

The masses in this partition function corresponding to (43) are given by

$$
\begin{array}{ll}
z_{1}=n \mu_{c}+n \mu, & z_{2}=n \mu_{c}-n \mu, \\
\bar{z}_{2}=n \mu-n \mu_{c}, & \bar{z}_{1}=-n \mu-n \mu_{c} .
\end{array}
$$

Substituting them into (87) we obtain

$$
Z_{2 \mid 2}=1-\frac{\mu^{2}}{\mu_{c}^{2}}+\frac{\mu^{2}}{\mu_{c}^{2}} e^{-4 N_{c} n \mu}
$$

which is exactly the microscopic limit of the quenched average phase factor (43) given in Eq. (44).

\section{DIRAC SPECTRUM}

We consider the Dirac operator (4) in a gauge where all gauge fields except $U_{n 1} \equiv U$ and $U_{1, n}=U^{\dagger}$ are equal to unity. If the eigenvalues of $U$ are equal to $\exp \left(i \theta_{k}\right)$ the eigenvalues of the Dirac operator are given by

$$
\lambda_{k, l}=\frac{1}{2}\left(e^{\frac{2 \pi i(k+1 / 2)+i \theta_{l}}{n}+\mu}-e^{-\frac{2 \pi i(k+1 / 2)-i \theta_{l}}{n}-\mu}\right), \quad k=1, \cdots, n, \quad l=1, \cdots, N_{c} .
$$

Contrary to $\mathrm{QCD}$ at $\mu \neq 0$ in more than one dimension, where the eigenvalues are scattered in the complex plane $[27,30,58,59,60,61]$, the eigenvalues are located on an ellipse in the complex plane with real and imaginary parts related by

$$
\left(\frac{\operatorname{Re}\left(\lambda_{k, l}\right)}{e^{\mu}-e^{-\mu}}\right)^{2}+\left(\frac{\operatorname{Im}\left(\lambda_{k, l}\right)}{e^{\mu}+e^{-\mu}}\right)^{2}=1
$$




\section{A. Universal Fluctuations}

In section $\amalg \mathrm{B}$ we have shown that the continuum limit of the staggered Dirac operator in one dimension is in the same universality class as QCD in three dimensions. The continuum limit of the staggered three dimensional Dirac operator, though, is in the chiral symmetry universality class of QCD in four dimensions. For example, in 62 this was found for the distribution of the small Dirac eigenvalues. The reason for the chiral structure is that the staggered Dirac operator only couples even and odd lattice sites. This is also the case in one dimension, but the off-diagonal blocks, containing the gauge fields, are the same in the continuum limit (or occur in the combination $U^{n}+U^{\dagger n}$ ), resulting in a two flavor theory with opposite masses (see (12)). The secular equation is given by

$$
\operatorname{det}\left[\left(\partial_{0}+i A_{0}+\mu+\lambda\right)\left(\partial_{0}+i A_{0}+\mu-\lambda\right)\right]=0 .
$$

This corresponds to the superposition of the spectrum of $\partial_{0}+A_{0}+\mu$ and $-\partial_{0}-i A_{0}-\mu$. In the domain of the Dirac spectrum where $\partial_{0}$ can be neglected, the eigenvalues on each of the lines $\pm \mu$ are given by the Hermitean random matrix ensemble $A_{0}$. For $\mu=0$ we have the superposition of the Hermitean random matrix ensembles of $A_{0}$ and $-A_{0}$. From the eigenvalues (90) it is clear that the staggered lattice Dirac eigenvalues show a similar superposition. One ensemble is localized on the left half of the ellipse and the other ensemble on its right half. Superpostions of more ensembles arise for correlations on scales larger than $N_{c}$ level spacings.

Eigenvalues of $U\left(N_{c}\right)$ matrices are correlated according to the circular unitary ensemble. Therefore, the eigenvalues $\lambda_{k, l}$ are correlated according the Gaussian Unitary Ensemble (GUE) on a scale where the variations in the average spectral density can be neglected, i.e. on a scale much less than $N_{c}$ level spacings. By correcting for the variation of the average level spacing, the scale on which universal random matrix correlations are found can be extended, but this scale should always remain well below $N_{c}$ level spacings. Then we are necessarily within one of the $n$ successive copies of the eigenvalues along the ellipse. At a scale of a finite number of average level spacings, we expect convergence to universal random matrix correlations in the large $N_{c}$ limit.

As measure of the correlations between eigenvalues, we use the number variance $\Sigma^{2}(\bar{n})$ defined as the variance on the number of eigenvalues in an interval that contains $\bar{n}$ eigenvalues on average. We calculate the number variance for intervals starting from zero. Because of the superposition of an enemble and its negative, this is equal to the number variance of a single ensemble for an interval that is symmetric about zero which is given by the GUE result. In Fig. 7 we show the number variance obtained from an average over $10^{5}$ gauge field configurations for $N_{c}=100$ and $\mu=0$. The number variance is calculated for an interval starting at zero. The discrepancy between the analytical result and the GUE (solid curve) is only barely visible.

The increase of the domain of validity of chiral random matrix theory with increasing $N_{c}$ was also observed for Dirac operator of QCD in four dimensions [63]. This can be explained as follows: The number of eigenvalues that is described by random matrix theory is equal to $\approx F_{\pi}^{2} \sqrt{V}$ (with $V$ the space-time volume), but $F_{\pi}^{2}$ scales with $N_{c}$ in the large $N_{c}$ limit.

\section{B. Chiral Symmetry Breaking at $\mu \neq 0$}

We first discuss the case of $\mu=0$. Then the average spacing of the eigenvalues scales as $1 /\left(n N_{c}\right)$. This means that the chiral condensate develops a discontinuity at $m=0$ when $n N_{c} \rightarrow \infty$. Two cases of interest where a nonzero chiral condensate can be obtained are the limit $n \rightarrow \infty$ for fixed $N_{c}$, i.e. at zero temperature, or at fixed $n$ in the limit $N_{c} \rightarrow \infty$. In both cases the chiral condensate is discontinuous for $n N_{c} \rightarrow \infty$ when the quark mass crosses the imaginary axis where the eigenvalues are located. However, Goldstone bosons and universal behavior is only found in the large $N_{c}$ limit. At fixed $N_{c}$, chiral symmetry breaking is not associated with universal behavior.

For $\mu \neq 0$ the Dirac eigenvalues are located on the ellipse (91). When the quark mass is inside the ellipse of eigenvalues the chiral condensate is zero for $n N_{c} \rightarrow \infty$ for the $S U\left(N_{c}\right)$ partition function and the phase quenched $U\left(N_{c}\right)$ partition function [24]. For gauge group $U\left(N_{c}\right)$ with $N_{f} \geq 1$ flavors, though, the partition function is $\mu$ independent so that the chiral condensate is also nonzero if the mass is inside the eigenvalue ellipse. The Banks-Casher formula fails in this case which is known as the "Silver Blaze Problem" [64]. The resolution is the same as for QCD in four dimensions [31]: the re-weighted eigenvalue distribution defined as

$$
\rho^{\mathrm{full}}(z)=\frac{1}{Z_{N_{f}}} \int_{U\left(N_{c}\right)} d U \operatorname{det}^{N_{f}} D \sum_{k} \delta^{2}\left(z-\lambda_{k}\right)
$$

shows oscillations with an amplitude that diverges exponentially with $n$ and a period that is proportional to $1 / n$. Below we will illustrate this for $U(1)$. 
For $N_{c}=1$ one easily derives that the spectral density is given by

$$
\rho^{\text {full }}(z)=4 \frac{e^{n \mu_{c}}+e^{-n \mu_{c}}-e^{n(i \alpha+\mu)}-e^{-n(i \alpha+\mu)}}{\left(e^{n \mu_{c}}+e^{-n \mu_{c}}\right)\left(e^{2 r}+e^{-2 r}+e^{2 i \alpha}+e^{-2 i \alpha}\right)} \delta(r-\mu),
$$

where $z$ is parameterized as

$$
z=\frac{1}{2}\left(e^{r+i \alpha}-e^{-r-i \alpha}\right), \quad r>0, \alpha \in[0,2 \pi]
$$

and we have used that $Z_{N_{f}=1}=2 \cosh \left(n \mu_{c}\right)$ (see eq. (8) ). For $\mu>\mu_{c}$ this spectral density has oscillations with an amplitude that diverges exponentially with $n$ and a period of order $1 / n$ which are the essential properties of the spectral density of the Dirac operator of QCD in four dimensions [31, 65, 66]. It can be decomposed as

$$
\rho^{\mathrm{full}}(z)=\rho^{\mathrm{q}}(z)+\rho^{\mathrm{osc}}(z)
$$

The average quenched spectral density is defined by

$$
\rho^{\mathrm{q}}(z)=\frac{1}{2 \pi} \int d \theta \frac{1}{n} \sum_{k=1}^{n} \delta^{2}\left(z-\lambda_{k}\right)
$$

with the eigenvalues $\lambda_{k}$ given by Eq. (90). After changing variables according to (95) and integrating over $\theta$ we find

$$
\rho^{\mathrm{q}}(z)=\frac{1}{2 \pi} \frac{4}{\left(e^{2 r}+e^{-2 r}+e^{2 i \alpha}+e^{-2 i \alpha}\right)} \delta(r-\mu) .
$$

The oscillatory part of the spectral density, $\rho^{\text {osc }}(z)$ is equal to the difference $\rho^{\text {full }}(z)-\rho^{\mathrm{q}}(z)$.

The chiral condensate is given by

$$
\Sigma^{\text {full }}(m)=\int d^{2} z \frac{\rho^{\text {full }}(z)}{z+m}
$$

and can also be decomposed as

$$
\Sigma^{\text {full }}(m)=\Sigma^{\mathrm{q}}(m)+\Sigma^{\mathrm{osc}}(m)
$$

The Jacobian for the transformation of $d^{2} z$ to $d r d \alpha$ is given by

$$
d^{2} z=\frac{1}{4}\left(e^{2 r}+e^{-2 r}+e^{2 i \alpha}+e^{-2 i \alpha}\right) d r d \alpha
$$

so that the chiral condensate after integration over $r$ can be simplified to

$$
\Sigma^{\text {full }}(m)=\frac{1}{\pi} \int_{0}^{2 \pi} d \alpha \frac{e^{n \mu_{c}}+e^{-n \mu_{c}}-e^{n(i \alpha+\mu)}-e^{-n(i \alpha+\mu)}}{\left(e^{n \mu_{c}}+e^{-n \mu_{c}}\right)\left(e^{\mu+i \alpha}-e^{-\mu-i \alpha}-2 m\right)}=\frac{\tanh \left(n \mu_{c}\right)}{\cosh \mu_{c}}
$$

This result remains finite for $n \rightarrow \infty$. We remind the reader that the mass is parameterized as $m=\sinh \mu_{c}$. This expression represents the resolvent at the quark mass which was evaluated in [49] and is in agreement with earlier work [6, 24]. Decomposing the spectral density and the chiral condensate according to (96) we obtain

$$
\begin{aligned}
\Sigma^{\mathrm{osc}}(m) & =\theta(\sinh (\mu)-m) \Sigma^{\mathrm{full}}(m), \\
\Sigma^{\mathrm{q}}(m) & =\theta(m-\sinh (\mu)) \Sigma^{\text {full }}(m),
\end{aligned}
$$

so that when the quark mass is inside the ellipse of eigenvalues, the entire chiral condensate is due to the oscillatory part of the spectral density. The discontinuity in the chiral condensate is reminiscent to a Stokes phenomenon. The alternative to the Banks-Casher relation proposed in [31] is also at work for QCD in one dimension. This solves the "Silver Blaze Problem" [64]. 


\section{CONCLUSIONS}

We have studied QCD in one dimension at nonzero chemical potential. Both the full theory, its quenched and phase quenched versions and gauge groups $U\left(N_{c}\right)$ and $S U\left(N_{c}\right)$ have been considered. In one dimension, the QCD or QCD-like partition functions can be reduced to a single matrix integral, which because of recent advances by Conrey, Farmer and Zirnbauer, could be evaluated analytically. In this paper we have analyzed the small mass behavior of the partition function, the nature of the sign problem, and the relation between the Dirac spectrum and chiral symmetry breaking.

To put our results in perspective, we emphasize that QCD in one dimension, is quite different from QCD in more dimensions. In particular, we wish to mention the following three points. First, instead of being scattered in the complex plane, the eigenvalues of the staggered Dirac operator are located on an ellipse in the complex plane. Second, phase transitions can only take place for zero temperature or for $N_{c} \rightarrow \infty$. At the critical chemical potential, a transition from the vacuum state to a state of free quarks takes place. Third, color singlets are made out of noninteracting quarks so that the critical chemical potential is given by the quark mass. In particular, the critical chemical potential for the meson state and the baryon state is the same.

Because the Dirac eigenvalues are located on a curve, the large $N_{c}$ limit of staggered lattice QCD in one dimension is in the same chiral symmetry class as QCD in three dimensions. We have shown this both by an explicit evaluation of the partition function, and by analyzing the fluctuations of the Dirac eigenvalues for large $N_{c}$. We have used this equivalence to explain the behavior of the partition functions and the average phase factor in the microscopic limit.

Contrary to QCD in four dimensions, the sign problem for QCD in one dimension is not severe in the case of gauge group $S U\left(N_{c}\right)$. One reason is that the critical chemical potential for full QCD and phase quenched QCD is the same, so that the parameter domain $m_{\pi} / 2<\mu<m_{N} / 3$, where the sign problem becomes severe in four dimensions, is absent in one dimension. A second reason is that for $\mu>\mu_{c}$ both the full theory and the phase quenched theory become a theory of free quarks with the same free energy in the thermodynamic limit.

For gauge group $U\left(N_{c}\right)$, on the other hand, the sign problem is severe when $\mu>m_{\pi} / 2$ both in one dimension and in four dimensions. The reason is that the $U\left(N_{c}\right)$ theory does not have charged excitations, whereas the phase quenched theory has charged mesons resulting in a phase transition at $\mu=m_{\pi} / 2$. We have evaluated the average phase factor both by averaging with respect to the phase quenched partition function and the full partition function, and similar conclusions have been reached.

The condititon $\mu>m_{\pi} / 2$ for having a severe sign problem can be rephrased as the quark mass being inside the ellipse of eigenvalues. In more dimensions this condition is that the quark mass is inside the support of the Dirac spectrum. It also applies to nonzero temperature as we have demonstrated explicitly in the framework of a chiral random matrix model.

Also for $U\left(N_{c}\right)$ QCD in one dimension, the chiral condensate is discontinuous across the imaginary axis in spite of the fact that there are no Dirac eigenvalues. This can only mean that the phase of the fermion determinant is responsible for the discontinuity. This is what happens in four dimensions where the discontinuity is due to a contribution to the spectral density that oscillates with a period of the inverse volume and amplitude that diverges exponentially with the volume. Exactly the same mechanism, where the discontinuity in the chiral condensate arises due to a Stokes like phenomenon, is at work in one dimension. This suggests that this is a universal mechanism for theories with a sign problem.

We end by repeating that QCD in one dimension with $S U\left(N_{c}\right)$ as gauge group has no serious sign problem. Our hope is that part of this conclusion translates to four dimensions ameliorating the sign problem for $\mu>M_{N} / 3$.

Acknowledgments. We wish to thank K. Splittorff for numerous comments and criticism and P.H. Damgaard, H. Neuberger and R.Pisarski are thanked for valuable discussions. K. Splittorff is also thanked for a careful reading of the manuscript. This work was supported by U.S. DOE Grant No. DE-FG-88ER40388 (JV), the Angelo Della Riccia Foundation (LR), the Villum Kann Rassmussen Foundation (JV) and the Danish National Bank (JV).

\section{APPENDIX A: AVERAGE $U\left(N_{c}\right)$ PHASE FACTOR FOR $N_{f}=1$}

In this appendix we give explicit results for the average phase factor for $N_{f}=1$ defined by

$$
\left\langle e^{2 i \theta}\right\rangle_{N_{f}=1}=\frac{Z_{2 \mid 1^{*}}\left(\mu_{c}, \mu\right)}{Z_{N_{f}=1}\left(\mu_{c}, \mu\right)} .
$$

The partition function $Z_{N_{f}=1}\left(\mu_{c}, \mu\right)$ was already given in Eq. (8). The numerator can be obtained from the CFZ formula. After taking the limit of degenerate critical chemical potentials at the end of the calculation, we obtain for 
$\mu<\mu_{c}$

$$
\begin{aligned}
Z_{2 \mid 1^{*}}\left(\mu_{c}, \mu\right)= & e^{n\left(N_{c}+1\right) \mu_{c}} e^{-4 n \mu} \frac{\left(1-e^{2 n\left(\mu+\mu_{c}\right)}\right)^{2}\left(1-e^{2 n\left(\mu-\mu_{c}\right)}\right)^{2}}{\left(e^{n \mu_{c}}-e^{-n \mu_{c}}\right)^{5}}+e^{-3 n\left(N_{c}+1\right) \mu_{c}} \frac{\left(e^{n \mu}-e^{-n \mu}\right)^{4}}{\left(e^{n \mu_{c}}-e^{-n \mu_{c}}\right)^{5}} \\
& +e^{-n\left(N_{c}+1\right) \mu_{c}}\left[f_{0}+N_{c} f_{1}+N_{c}^{2} f_{2}\right] .
\end{aligned}
$$

with

$$
\begin{aligned}
f_{0}= & \frac{2 e^{2 n\left(\mu+\mu_{c}\right)}+2 e^{2 n\left(\mu-\mu_{c}\right)}-e^{4 n \mu}-e^{-4 n \mu}+4 e^{2 n \mu}+4 e^{-2 n \mu}-e^{4 n\left(\mu-\mu_{c}\right)}-e^{4 n\left(\mu+\mu_{c}\right)}+2 e^{6 n \mu_{c}-2 n \mu}+2 e^{6 n \mu_{c}+2 n \mu}}{\left(e^{n \mu_{c}}-e^{-n \mu_{c}}\right)^{5}} \\
& +\frac{-10 e^{2 n \mu_{c}}+6 e^{4 n \mu_{c}}-2-5 e^{6 n \mu_{c}}-e^{-2 n \mu_{c}}}{\left(e^{n \mu_{c}}-e^{-n \mu_{c}}\right)^{5}}, \\
f_{1}= & \frac{\left(1-e^{-2 n \mu}\right)^{2}\left(3 e^{2 n \mu_{c}}+e^{-2 n \mu_{c}}-2 e^{2 n \mu}-2 e^{-2 n \mu}\right)}{\left(e^{n \mu_{c}}-e^{-n \mu_{c}}\right)^{3}}, \\
f_{2}= & -\frac{\left.\left.\left(1-e^{-2 n \mu}\right)^{2}\left(1-e^{n\left(\mu+\mu_{c}\right.}\right)\right)\left(1-e^{n\left(\mu-\mu_{c}\right.}\right)\right)}{\left(e^{n \mu_{c}}-e^{-n \mu_{c}}\right)^{3}} .
\end{aligned}
$$

For $\mu>\mu_{c}$ a similar calculation results in

$Z_{2 \mid 1^{*}}=N_{c} e^{n N_{c}\left(\mu_{c}-2 \mu\right)} \frac{\left(1-e^{-2 n\left(\mu+\mu_{c}\right)}\right)\left(1-e^{-2 n \mu}\right)}{\left(1-e^{-2 n \mu_{c}}\right)^{2}}\left(1+\frac{g_{1}}{N_{c}}\right)+N_{c} e^{-n N_{c}\left(2 \mu+\mu_{c}\right)} \frac{\left(1-e^{2 n\left(\mu_{c}-\mu\right)}\right)\left(1-e^{-2 n \mu}\right)}{\left(1-e^{2 n \mu_{c}}\right)^{2}}\left(1+\frac{g_{2}}{N_{c}}\right)$

with

$$
\begin{aligned}
& g_{1}=1+\frac{1}{e^{2 n \mu}-1}+\frac{1}{e^{2 n\left(\mu+\mu_{c}\right)}-1}-\frac{2}{e^{2 n \mu_{c}}-1}, \\
& g_{2}=1+\frac{1}{e^{2 n\left(\mu-\mu_{c}\right)}-1}+\frac{1}{e^{2 n \mu}-1}-\frac{2}{e^{-2 n \mu_{c}}-1}
\end{aligned}
$$

We have checked that the results in this appendix agree with the large $N_{c}$ limit of the average phase factor given in section IV B.

[1] M. P. Lombardo, arXiv:hep-lat/0612017

[2] C. Schmidt, PoS LAT2006, 021 (2006) arXiv:hep-lat/0610116.

[3] K. Splittorff, arXiv:hep-lat/0610072

[4] K. Splittorff and J. J. M. Verbaarschot, arXiv:hep-lat/0702011.

[5] K. Splittorff and J. J. M. Verbaarschot, Phys. Rev. Lett. 98, 031601 (2007) arXiv:hep-lat/0609076.

[6] P. E. Gibbs, Preprint PRINT-86-0389-GLASGOW, 1986; P. E. Gibbs, Phys. Lett. B 182 (1986) 369.

[7] D. Toussaint, Nucl. Phys. Proc. Suppl. 17, 248 (1990).

[8] P. de Forcrand and V. Laliena, Phys. Rev. D 61, 034502 (2000) arXiv:hep-lat/9907004.

[9] Y. Sasai, A. Nakamura and T. Takaishi, Nucl. Phys. Proc. Suppl. 129, 539 (2004) arXiv:hep-lat/0310046.

[10] S. Ejiri, Phys. Rev. D 69, 094506 (2004) arXiv:hep-lat/0401012; S. Ejiri, Phys. Rev. D 73, 054502 (2006) arXiv:hep-lat/0506023.

[11] C. Schmidt, Z. Fodor and S. D. Katz, arXiv:hep-lat/0512032

[12] C. R. Allton et al., Phys. Rev. D 66, 074507 (2002) arXiv:hep-lat/0204010; C. R. Allton, S. Ejiri, S. J. Hands, O. Kaczmarek, F. Karsch, E. Laermann and C. Schmidt, Phys. Rev. D 68, 014507 (2003) arXiv:hep-lat/0305007; C. R. Allton et al., Phys. Rev. D 71, 054508 (2005) arXiv:hep-lat/0501030.

[13] E. V. Shuryak and J. J. M. Verbaarschot, Nucl. Phys. A 560, 306 (1993) arXiv:hep-th/9212088.

[14] J. J. M. Verbaarschot, Phys. Rev. Lett. 72, 2531 (1994) arXiv:hep-th/9401059.

[15] J.B. Conrey, D.W. Farmer and M.R. Zirnbauer, arXiv:math-ph/0511024], 2005.

[16] E. M. Ilgenfritz and J. Kripfganz, Z. Phys. C 29, 79 (1985).

[17] P. H. Damgaard, N. Kawamoto and K. Shigemoto, Phys. Rev. Lett. 53 (1984) 2211; Nucl. Phys. B 264, 1 (1986).

[18] A. Gocksch and M. Ogilvie, Phys. Rev. D 31 (1985) 877.

[19] P. H. Damgaard, D. Hochberg and N. Kawamoto, Phys. Lett. B 158 (1985) 239.

[20] N. Bilic, K. Demeterfi and B. Petersson, Nucl. Phys. B 377, 651 (1992).

[21] P. de Forcrand and S. Kim, arXiv:hep-lat/0608012 
[22] P. E. Gibbs, Phys. Lett. B 172, 53 (1986).

[23] A. Gocksch, Phys. Rev. D 37, 1014 (1988).

[24] N. Bilic and K. Demeterfi, Phys. Lett. B 212, 83 (1988).

[25] S. Gupta, Phys. Lett. B 588, 136 (2004) arXiv:hep-lat/0307007.

[26] M. P. Lombardo, Nucl. Phys. Proc. Suppl. 83, 375 (2000) arXiv:hep-lat/9908006.

[27] I. Barbour, N. E. Behilil, E. Dagotto, F. Karsch, A. Moreo, M. Stone and H. W. Wyld, Nucl. Phys. B 275, 296 (1986).

[28] J. J. M. Verbaarschot and I. Zahed, Phys. Rev. Lett. 73, 2288 (1994) arXiv:hep-th/9405005.

[29] A. J. McKane and M. Stone, Annals Phys. 131, 36 (1981).

[30] M. A. Stephanov, Phys. Rev. Lett. 76, 4472 (1996) arXiv:hep-lat/9604003.

[31] J. C. Osborn, K. Splittorff and J. J. M. Verbaarschot, Phys. Rev. Lett. 94, 202001 (2005) arXiv:hep-th/0501210.

[32] P. Hasenfratz and F. Karsch, Phys. Lett. B 125, 308 (1983).

[33] M.R. Zirnbauer, arXiv:chao-dyn/9609007.

[34] B. Schlittgen and T. Wettig, Nucl. Phys. B 632, 155 (2002) arXiv:hep-lat/0111039.

[35] J. Budczies, S. Nonnenmacher, Y. Shnir and M. R. Zirnbauer, Nucl. Phys. B 635, 309 (2002) arXiv:hep-lat/0112018.

[36] J.B. Conrey, D.W. Farmer, J.P. Keating, M.O. Rubinstein and N.C. Snaith, arXiv:math-ph/0208007.

[37] E.L. Basor and P.J. Forrester, Mathematische Nachrichten, 170, 5 (1194).

[38] Z. Fodor and S. D. Katz, JHEP 0203, 014 (2002) arXiv:hep-lat/0106002.

[39] Z. Fodor and S. D. Katz, JHEP 0404, 050 (2004) arXiv:hep-lat/0402006.

[40] P. de Forcrand and O. Philipsen, Nucl. Phys. B 642, 290 (2002) arXiv:hep-lat/0205016].

[41] P. de Forcrand and O. Philipsen, Nucl. Phys. B 673, 170 (2003) arXiv:hep-lat/0307020.

[42] M. D'Elia and M. P. Lombardo, Phys. Rev. D 67, 014505 (2003) arXiv:hep-lat/0209146].

[43] C. R. Allton, S. Ejiri, S. J. Hands, O. Kaczmarek, F. Karsch, E. Laermann and C. Schmidt, Phys. Rev. D 68, 014507 (2003) arXiv:hep-lat/0305007.

[44] C. R. Allton et al., Phys. Rev. D 71, 054508 (2005) arXiv:hep-lat/0501030].

[45] R. V. Gavai and S. Gupta, Phys. Rev. D 68, 034506 (2003) arXiv:hep-lat/0303013.

[46] R. V. Gavai and S. Gupta, Phys. Rev. D 71, 114014 (2005) arXiv:hep-lat/0412035.

[47] V. Azcoiti, G. di Carlo and A. F. Grillo, Phys. Rev. Lett. 65, 2239 (1990); J. Ambjorn, K. N. Anagnostopoulos, J. Nishimura and J. J. M. Verbaarschot, JHEP 0210, 062 (2002) arXiv:hep-lat/0208025.

[48] A. D. Jackson and J. J. M. Verbaarschot, Phys. Rev. D 53, 7223 (1996) arXiv:hep-ph/9509324].

[49] M. A. Halasz, A. D. Jackson and J. J. M. Verbaarschot, Phys. Rev. D 56 (1997) 5140 arXiv:hep-lat/9703006].

[50] K. Splittorff, arXiv:hep-lat/0505001

[51] G. V. Dunne and S. M. Nishigaki, Nucl. Phys. B 670, 307 (2003) arXiv:hep-ph/0306220.

[52] G. Akemann and P. H. Damgaard, Nucl. Phys. B 576, 597 (2000) arXiv:hep-th/9910190.

[53] P. H. Damgaard and S. M. Nishigaki, Phys. Rev. D 57, 5299 (1998) arXiv:hep-th/9711096.

[54] G. Akemann, D. Dalmazi, P. H. Damgaard and J. J. M. Verbaarschot, Nucl. Phys. B 601, 77 (2001) arXiv:hep-th/0011072.

[55] T. Andersson, P. H. Damgaard and K. Splittorff, Nucl. Phys. B 707, 509 (2005) arXiv:hep-th/0410163.

[56] G. Akemann and Y. V. Fyodorov, Nucl. Phys. B 664, 457 (2003) arXiv:hep-th/0304095.

[57] R. J. Szabo, Nucl. Phys. B 598, 309 (2001) arXiv:hep-th/0009237.

[58] D. Toublan and J. J. M. Verbaarschot, Int. J. Mod. Phys. B 15, 1404 (2001) arXiv:hep-th/0001110.

[59] K. Splittorff and J. J. M. Verbaarschot, Nucl. Phys. B 683, 467 (2004) arXiv:hep-th/0310271.

[60] G. Akemann and T. Wettig, Phys. Rev. Lett. 92, 102002 (2004) [Ibid. 96, 029902 (2006)] arXiv:hep-lat/0308003].

[61] J. Bloch and T. Wettig, Phys. Rev. Lett. 97, 012003 (2006) arXiv:hep-lat/0604020.

[62] P. H. Damgaard, U. M. Heller, A. Krasnitz and T. Madsen, Phys. Lett. B 440, 129 (1998) arXiv:hep-lat/9803012.

[63] R. Narayanan and H. Neuberger, Nucl. Phys. B 696, 107 (2004) arXiv:hep-lat/0405025.

[64] T. D. Cohen, Phys. Rev. Lett. 91, 222001 (2003) arXiv:hep-ph/0307089.

[65] J. C. Osborn, Phys. Rev. Lett. 93, 222001 (2004) arXiv:hep-th/0403131.

[66] G. Akemann, J. C. Osborn, K. Splittorff and J. J. M. Verbaarschot, Nucl. Phys. B 712, 287 (2005) arXiv:hep-th/0411030. 